\title{
ArcheoSciences
}

Revue d'archéométrie

\section{L'outil multidimensionnel dans l'analyse spatiale en milieu lacustre : l'exemple des habitats d'Egolzwil 3 (Ch) et de Charavines-les-Baigneurs B3 (F)}

\section{Claire Tardieu}

\section{OpenEdition}

\section{Journals}

Édition électronique

URL : https://journals.openedition.org/archeosciences/628

DOI : 10.4000/archeosciences.628

ISBN : 978-2-7535-1594-9

ISSN : 2104-3728

\section{Éditeur}

Presses universitaires de Rennes

\section{Édition imprimée}

Date de publication : 31 décembre 2005

Pagination : 133-150

ISSN : 1960-1360

\section{Référence électronique}

Claire Tardieu, «L'outil multidimensionnel dans l'analyse spatiale en milieu lacustre : I'exemple des habitats d'Egolzwil 3 (Ch) et de Charavines-les-Baigneurs B3 (F) », ArcheoSciences [En ligne], 29 | 2005, mis en ligne le 31 décembre 2007, consulté le 01 février 2022. URL : http://journals.openedition.org/ archeosciences/628; DOI : https://doi.org/10.4000/archeosciences.628 


\title{
L'outil multidimensionnel dans l'analyse spatiale en milieu lacustre : l'exemple des habitats d'Egolzwil 3 (Ch) et de Charavines-les- Baigneurs B3 (F)
}

\author{
Claire TARDIEU*
}

\begin{abstract}
Résumé : Le milieu lacustre recèle des potentialités exceptionnelles pour aborder les caractères socio-économiques des communautés de bords de lac durant le Néolithique. L'organisation et le fonctionnement villageois se reflètent dans les distributions horizontales des vestiges. Toutefois la présence du lac, les conditions de conservation et les structures architecturales soulèvent des problèmes très spécifiques auxquels les techniques multidimensionnelles proposent une solution. Cela suppose, de s'affranchir (au moins dans un premier temps) des structures architecturales pour se baser uniquement sur la répartition au sol des témoins.

Les exemples d'application de la méthode sur les stations d'Egolzwil 3 (Lucerne, Suisse), ct de Charavines-les-Baigneurs (Isère, France), illustrent des exploitations possibles face aux caractéristiques du milieu lacustre (nappes quasi continues de vestiges, champs de picux, grandes surfaces excavées...). Dans ces stations, les répartitions horizontales des témoins unc fois démélées ont révélé une organisation clairc de l'espace liće aux activités. Ainsi, s'identifient sous l'auvent des activités artisanales variées, autour des foyers intéricurs des zones domestiques et d'un point de vue plus global, des unités d'habitations présentant des indices de fonctionnements différents.
\end{abstract}

\begin{abstract}
Lakeshore settlements contain potentially exceptional data relating to socio-economic aspects of populations occupying lakesides during the Neolithic. The horizontal distribution of archacological remains show how villages were organized and how they functioned. However, the lakes, site preservation and architectural features raise quite specific problems which can be solved by multidimensional methods. To this end, it was necessary to put aside the architectural features (at least initially) and restrict the analysis to the distribution of archacological remains on the ground.

Faced with the specific problems of lakeshore settlements (near continuous distribution of finds, fields of piles, extensive excavation areas...), the application of this method to the sites of Egolzwil 3 (Switzerland), and Charavines-les-Baigneurs (Isère, France) shows that solutions are possible.

In these settlements, analysis of horizontal distributions of archacological remains reveals clear patterning, showing craft activities in the interior of houses around hearths, as well as evidence for differential functioning of houses within the villages.
\end{abstract}

Mots-clés : Organisation spatiale, lacustre, analyse des données, Néolithique

Key-words: Intrasite spatial analysis, lakeshore settlement, multivariate analysis, Neolithic.

\section{Introduction}

La question de l'organisation et du fonctionnement des communautés néolithiques est un thème récurrent dans les études archéologiques, dont la dimension spatiale constitue un moyen d'accès privilégié. Les villages de bords de lacs ont longtemps suscité l'envie en raison de leur conservation exceptionnelle des vestiges organiques. Mais, même lorsque est connue avec précision l'implantation et la dynamique des structures architecturales, la question de l'appropriation de l'espace demeure confuse. Comment étaient organisés les espaces domestiques et villageois. Quels sont leurs liens et leurs rôles?

Notre but est ici de chercher à comprendre comment les hommes qui occupaient les rives de bords de lacs dans le domaine circum-alpin durant le Néolithique avaient organisé leurs espaces de vie. Toutefois, si le milieu lacustre peut apporter des clefs de compréhension exceptionnelles, il n'en écarte pas pour autant les difficultés d'un traitement spatial comme la durée des formations des couches et soulève également des problèmes spécifiques, allant de la définition d'un niveau d'occupation au biais taphonomiques liés à la présence de l'eau.

Nous énoncerons ainsi des questions archéologiques soulevées par ces problématiques spatiales particulières et amenant à l'emploi d'outils statistiques multidimensionnels, et présenterons les principes généraux d'application de ces outils.

\footnotetext{
* Maison de l'archéologie et de l'ethnologie, UMR 704I - Protohistoire européenne - 21, allée de l'université, 92023 NANTERRE cedex. ctardieu@mae.u-parisI0.fr
} 


\section{Cadre de l'étude}

\section{A. Spécificités du milieu lacustre dans le cadre d'une approche spatiale}

L'examen de l'évolution du climat montre l'étroite relation existant entre les habitats littoraux néolithiques et leur bassin lacustre (Magny, 1993). En effet, l'installation d'un groupe se produit principalement durant les périodes de basses eaux, lors d'une phase régressive. A l'inverse, les villages disparaissent lors des phases transgressives où le niveau de l'eau remonte. Ces phases d'occupations et d'abandons liées aux niveaux de l'eau ne signifient ni l'absence d'inondations dans les villages de bas rivages, ni une causalité unique aux occupations cycliques (Pétrequin, Arbogast et al., 2002). En outre, le village pouvait être déplacé en direction de la terre ferme à l'occasion de crues plus fortes durant quelques années (Pétrequin et Pétrequin, 1984). La forte interaction existant entre l'homme et son milieu pousse à considérer le lac comme un élément essentiel des répartitions de vestiges au sol.

Cette proximité des hommes et de l'eau a conduit à des aménagements spécifiques: structures architecturales surélevées, élaboration de zones surélevées, consolidation du sol non praticable, chemin d'accès... Le lac agit donc comme un agent de la formation des répartitions de témoin.

Seuls ou associés à d'autres vestiges, les restes organiques permettent d'envisager des aspects socio-économiques rares : réserves de grains, lieux de consommation alimentaire, espaces de stockage... Toutefois, ni la variété ni la quantité des témoins ne doivent occulter le fait que, comme dans les sites terrestres, nous ne pouvons accéder qu'à des données tronquées et non à la totalité des vestiges. De plus, les témoins des activités des hommes sont souvent inégalement conservés ; toutes les activités ne laissent, en effet, pas les mêmes traces et toutes les traces ne sont pas conservées de la même façon.

Les brèves occupations des villages, les déplacements réguliers du centre du village, et les abandons parfois précipités par la remontée du niveau de l'eau ont laissé mêlé au sol des rejets et des vestiges encore en cours d'utilisation mais ne témoignent le plus souvent que d'une organisation. "C'est ainsi une surface reconnaissable sur laquelle a vécu l'homme ... pendant un laps de temps suffisamment court pour que l'on puisse espérer déduire de la position des vestiges quelque chose au sujet des activités " (Bordes, 1975, p. 139).

La richesse des sites lacustres présente aussi ses revers. Si la conservation des restes organiques permet de retrouver les poteaux des structures architecturales, ces dernières ne sont pas pour autant facilement identifiables. En effet, malgré les apports indéniables de la dendrochronologie un flou perdure dans certains plans. Cette confusion est à lier aux reconstructions, aux réparations, à la proximité entre les constructions tout cela faisant ainsi découvrir une forêt de poteaux lors de la fouille. Par ailleurs, les datations obtenues par dendrochronologie révèlent l'abattage des arbres et non les constructions des structures. Si la conservation exceptionnelle des témoins abandonnés ou des outils encore utilisés offre une grande variété mais aussi une grande quantité de témoins, l'étendue des nappes de vestiges est quasi continue. Cette continuité, accentuée par l'absence de structures en creux, place tous les vestiges sur un même plan horizontal, rendant délicate l'association entre ces nappes de vestiges et les structures architecturales correspondantes. De plus, selon la proximité du lac, la position des dépotoirs peut varier (Pétrequin et Pétrequin, 1984).

Si l'eau permet une bonne conservation, elle peut aussi perturber la formation des niveaux archéologiques et, de ce fait, l'organisation au sol des vestiges pendant l'occupation, lors du départ et après l'abandon. La surface peut avoir été agitée par des vagues et/ou des courants. Ces facteurs perturbateurs agissent sur les vestiges, provoquant des remaniements pouvant aller d'un simple flottage des éléments légers à une érosion complète des niveaux archéologiques. Les études de $\mathrm{M}$. Magny ont permis d'évaluer et de nuancer la notion de remaniement (Magny, 1978). Après l'occupation, l'intensité des bouleversements dépend de la remontée plus ou moins rapide du niveau de l'eau. Si la remontée est rapide, seule la partie superficielle de la couche sera touchée mais le cœur sera protégé des actions de l'eau, vagues ou courants. En revanche, si la sédimentation est lente, le battement des vagues et les courants auront plus longtemps l'occasion d'agir sur les vestiges. Des essais ont été réalisés pour classer les couches d'habitat sur une échelle de remaniement allant de l'action de détail (tessons roulés) au remaniement d'ensemble (lessivage complet) (Bocquet $\&$ Christien, 1993). Nous menons dans ce domaine une expérimentation visant à quantifier les déplacements des vestiges après leur abandon. Des perturbations par l'eau peuvent également se produire durant l'occupation, lors de remontées du niveau de l'eau. Enfin, n'oublions pas que des facteurs anthropiques ont pu disperser les vestiges après leur dépôt (piétinements, zones de circulation, actions animales...).

\section{B. Le milieu lacustre moins favorable que le milieu ter- restre?}

La clarté des distributions de vestiges sur des campements préhistoriques emblématiques à l'instar du site de Pincevent (Leroi-Gourhan et Brezillon, 1966) est malheureusement loin des répartitions au sol des vestiges d'autres sites même si sur certains sites «terrestres " ou en grotte, des approches spatiales ont tout de même dévoilé des organisations nettes. En effet, dès la fouille, des structures spatiales y sont apparues, révélant clairement les foyers, les zones de rejet, soit autour des foyers, soit de part et d'autre des habitations dans des fosses dépotoirs. Dans le village chalcolithique (Fontbouisse) de Boussargues (Argelliers, Hérault), c'est l'organisation des espaces intérieurs observée à partir des densités de vestiges matériels au sol, qui a montré une structuration fine avec des aires d'activités, des zones de stockage, de fabrication de poteries, de meunerie et de circulation (Colomer et al., 1990). Dans la grotte des Planches prèsArbois, l'étude de la zone naturellement abritée de l'occupation Bronze final (horizon C3), fait écho d'un parc à 
bétail, des espaces de stockage, des zones foyères semblables entre elles... (Pétrequin, 1981). En milieu lacustre, les tentatives sur les répartitions horizontales des vestiges sont encore rares et centrées sur des questions précises. Les approches spatiales dépendent de l'identification des structures architecturales, des difficultés à associer structures architecturales et couches archéologiques, de l'étendue des surfaces excavées...

\section{Quelles méthodes?}

Les contraintes archéologiques précédemment énoncées ne permettent que rarement de caractériser la dynamique architecturale et autorise encore moins à associer avec certitude un matériel à une structure architecturale, ce qui rend difficile d'appuyer le raisonnement spatial sur l'association vestiges-structures. Nous proposons une première étape d'analyse qui permettra de déceler la structuration de l'espace. L'analyse doit alors se baser uniquement sur la répartition des vestiges au sol. L'analyse spatiale devient alors la recherche des phénomènes comportementaux et des processus impliquant la formation des sols d'occupation (Djindjian, 1988). Le but est de favoriser l'identification archéologique de ces phénomènes structurels expliquant les distributions archéologiques et à partir desquels on peut espérer déduire des pratiques et des comportements sociaux culturels ou identitaires significatifs. Mes objectifs sont donc de rechercher les organisations spatiales générales, de repérer des zones particulières et d'établir des relations entre ces zones. Ces zones spécifiques peuvent être de tailles différentes, de formes variables et souvent irrégulières, de différentes densités et, bien sûr, de compositions variables (Whallon, 1973). Les formations de ces assemblages de témoins répondent à différents principes déjà détaillés par F. Djindjian (Djindjian, 1988).

Le choix d'une démarche analytique permet d'appréhender l'ensemble de l'espace fouillé et à partir d'une grande variété de témoins. En effet, elle permet de contrôler de nombreuses informations, y compris de natures différentes, et offre la possibilité de croiser l'ensemble des données et ainsi de déterminer des liaisons entre les différents phénomènes, même les plus marginaux. Elle n'est cependant que la première étape de l'analyse des distributions qui devra être poursuivie par une étape ou chaque zone isolée (aire d'activité) sera observée selon sa composition par catégorie typologique. Les aspects qualitatifs indispensables seront alors pris en considération (aspects technologiques de la chaîne opératoire, indice de spécialisation, topographie...). Enfin, nous tenons à rappeler que toute analyse ne peut être menée qu'après s'être assuré de l'absence de biais taphonomiques.

\section{Méthodes pour une analyse spatiale}

\section{A. Vers la dimension spatiale}

Si des fouilleurs commencent à enregistrer les données selon leur localisation $(\mathrm{x}, \mathrm{y}, \mathrm{z})$ dès le milieu du $\mathrm{XIX}^{\mathrm{c} m \mathrm{c}}$ siècle, cette pratique deviendra régulière qu'un siècle plus tard. Le milieu du $\mathrm{XX}^{\mathrm{cmc}}$ siècle, marquant l'exploitation progressive de l'enregistrement de l'emplacement des découvertes sera progressivement exploité, développant les techniques de localisation parallèlement aux besoins. «En fait de méthodes, d'ailleurs, on ne peut jamais faire que du provisoire car les méthodes changent à mesure que la science avance» (Durkheim, 1937).

Les statistiques descriptives font désormais partie intégrante des traitements de données en sciences humaines. L'archéologie y fait de plus en plus souvent appel en raison des séries de plus en plus nombreuses à traiter. Les méthodes d'analyse de données permettent une étude globale des individus et des variables. La recherche des ressemblances et/ou des différences entre les individus est un des objectifs de ces analyses : on considère que deux individus se ressemblent lorsque leurs profils selon les différents caractères sont voisins. L'analyse des données apparaît dans les années trente et voit son accomplissement surtout à partir des années soixante. Elle regroupe toutes les méthodes statistiques analysant la structure de données décrites par de très nombreuses variables. Ces méthodes consistent à réduire les dimensions d'un phénomène à un petit nombre et à fournir des paramètres d'explication à ces dimensions. L'analyse multidimensionnelle rend possible la représentation des distances statistiques entre les individus sur un graphique. En fonction des rôles joués par chaque caractère, on peut mettre en évidence des groupes de caractères, corrélés, dépendants et interdépendants. Les méthodes de traitement quantitatives impliquent un recours systématique à l'informatique. Ainsi, l'apparition des ordinateurs et leur " popularisation » a permis la vulgarisation des techniques statistiques de l'analyse de données. Nous travaillons sous $\underline{\text { Spad }}$ ' (développée par Decisia) car ce logiciel est accessible aux utilisateurs non mathématiciens et offre un rendu graphique convivial. L'emploi des outils statistiques pour des thématiques spatiales a commencé à se développer dès les années 60 . Nous en proposons, ci dessous, un récapitulatif (tab. 1).

Les fondements de la méthode utilisée sont développés par F. Djindjian (1988), sur la base des principes mis en place par R. Whallon, l' « Unconstrained Clustering » de 1984.

\section{B. La méthode de structuration spatiale de F. Djindjian (cf. fig. 1)}

Une démarche analytique permet une étude globale des individus et des variables, en utilisant généralement des représentations graphiques explicites. Elle met en œuvre des outils multidimensionnels consistant en une traduction des données de base en des données statistiques. Les traitements multidimensionnels, l'utilisation des composantes principales ou des facteurs d'analyse se basent sur les données calculées. Ils supposent l'existence de modèles de covariation, globaux et linéaires, parmi les types d'objets dispersés dans l'espace analysé (Whallon, 1984). La procédure de l'analyse factorielle

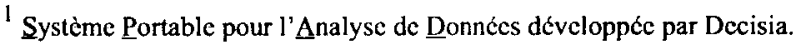




\begin{tabular}{|c|c|c|c|c|c|}
\hline PERIODES & Début 1960 & Fin 1960 & 1970 & 1975 & 1985 \\
\hline OBJECTIFS & $\begin{array}{l}\text { Déterminer les } \\
\text { perturbations } \\
\text { naturelles en } \\
\text { repérant si les } \\
\text { distributions } \\
\text { sont ordonnées }\end{array}$ & $\begin{array}{l}\text { Déterminer } \\
\text { d'éventuelles } \\
\text { spćcificités en } \\
\text { caractérisant les } \\
\text { distributions par } \\
\text { des associations } \\
\text { de vestiges }\end{array}$ & $\begin{array}{lr}\text { Mettre } & \text { en } \\
\text { évidence } & \text { la } \\
\text { variabilité } & \text { des } \\
\text { assemblages } & \end{array}$ & \begin{tabular}{|lr} 
Déterminer les \\
processus \\
formation des sites
\end{tabular} & $\begin{array}{l}\text { Appréhender } \\
\text { simultanément: } \\
\text { taille, forme, } \\
\text { composition, } \\
\text { densité et } \\
\text { étalement des } \\
\text { vestiges (Kroll et } \\
\text { Price 1991) }\end{array}$ \\
\hline METHODES & $\begin{array}{l}\text { ()bscrver les } \\
\text { tendances } \\
\text { répétitives dans } \\
\text { les répartitions }\end{array}$ & \begin{tabular}{|lr} 
Choisir & des \\
critères & descriptifs \\
précis autorisant \\
la mise & en \\
parallèle & \\
d'artefacts & de \\
natures & \\
différentes & \\
\end{tabular} & $\begin{array}{l}\text { Déterminer des } \\
\text { "tool kits" et } \\
\text { des aires } \\
\text { d'activités } \\
\text { structurant } \\
\text { l'espace }\end{array}$ & $\begin{array}{l}\text { Chercher les } \\
\text { facteurs à l'origine } \\
\text { du dépôt et les } \\
\text { phénomènes post- } \\
\text { dépositionnels. }\end{array}$ & $\begin{array}{lr}\text { Déterminer } & \text { les } \\
\text { critères } & \text { en } \\
\text { interaction pour } \\
\text { retenir ceux qui } \\
\text { agissent sur la } \\
\text { structuration de } \\
\text { l'espace }\end{array}$ \\
\hline $\begin{array}{l}\text { MOYENS MIS } \\
\text { EN CEUVRE }\end{array}$ & $\begin{array}{l}\text { Plans } \\
\text { composites }\end{array}$ & $\begin{array}{l}\text { Plans spécifiques } \\
\text { aux } \\
\text { caractéristiques } \\
\text { données }\end{array}$ & $\begin{array}{l}\text { Techniques } \\
\text { quantitatives }\end{array}$ & Ethnoarchéologie & $\begin{array}{l}\text { "Unconstrained } \\
\text { clustering" } \\
\text { structuration } \\
\text { spatiale }\end{array}$ \\
\hline $\begin{array}{c}\text { ET } \\
\text { AUTEURS }\end{array}$ & $\begin{array}{l}\text { - Clark \& Clark } \\
\text { (1954) } \\
\text { comprendre la } \\
\text { nature des aires } \\
\text { d'habitation } \\
\text { - Ieakey (1971) } \\
\text { concentrations } \\
\text { verticales, } \\
\text { associations } \\
\text { horizontales, } \\
\text { configurations } \\
\text { horizontales } \\
\text { spécifique afin } \\
\text { de définir des } \\
\text { (occupations } \\
\text { sites " et des } \\
\text { « living floors") }\end{array}$ & 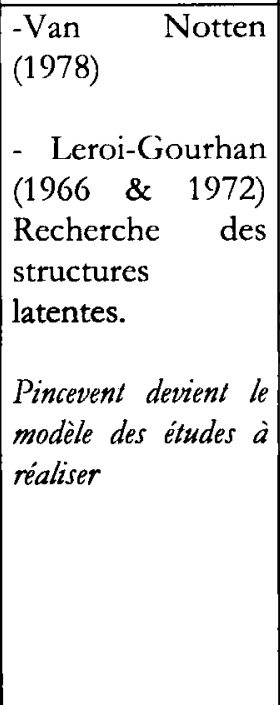 & $\begin{array}{l}\text { (1973) } \\
\text { Recherche des } \\
\text { distributions } \\
\text { non aléatoires et } \\
\text { spécifiques dans } \\
\text { des assemblages } \\
\text { moustériens }\end{array}$ & 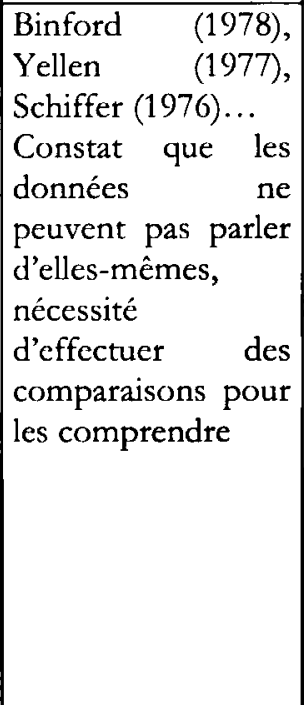 & $\begin{array}{l}\text { - Whallon (1984), } \\
\text { Djindjian (1988) } \\
\text { Traiter } \\
\text { simultanément de } \\
\text { grandes } \\
\text { distributions }\end{array}$ \\
\hline
\end{tabular}

Tableau 1 : Synthèse de l'évolution des problématiques et des méthodes d'analyse spatiales en archéologie.

Table 1: Synthesis of the principal points resolved in the archaeological researches in spatial analyses.

des correspondances ou des composantes principales permet de définir des groupes d'objets corrélés par l'utilisation d'une matrice de coefficients de corrélation ou d'un tableau disjonctif (Whallon, 1973). Une telle procédure permet, par l'observation des rôles joués par chaque caractère, la mise en évidence de groupes de caractères de témoins corrélés, dépendants ou s'excluant. Les moyens mis en œuvre vont permettre d'étudier la variation spatiale éventuelle d'une distribution, voire de plusieurs distributions simultanément, même si elles sont de natures très différentes, cela afin de repérer d'éventuelles associations/répulsions entre les témoins. Nous allons ainsi rechercher s'il existe des similarités entre les compositions des surfaces des unités de fouilles. Une difficulté réside alors dans la détermination du seuil de similarité. On peut ainsi mettre en évidence en fonction des rôles joués par chaque caractère des groupes de caractères dépendants, interdépendants ou corrélés.

Cet outil d'analyse s'applique à partir d'un tableau de comptage ou sur les unités de fouilles. La base établie pour chaque site afin de réaliser les analyses est un tableau d'effectif, unité de fouilles / catégorie de vestiges où, à l'intersection, figure l'effectif des individus de la catégorie présente dans cette unité. Nous avons observé la fouille: système technique, système d'enregistrement, échantillonnage. Les catégories de vestiges sous forme de comptage peuvent être indiqués en nombre ou en poids. La méthode se déroule en 6 étapes (d'après Djindjian, 1988).

\section{Lissage}

Un lissage est effectué sur les distributions de vestiges par moyennes concentriques. Il consiste en un calcul de moyennes à partir des huit unités de fouilles adjacentes. 


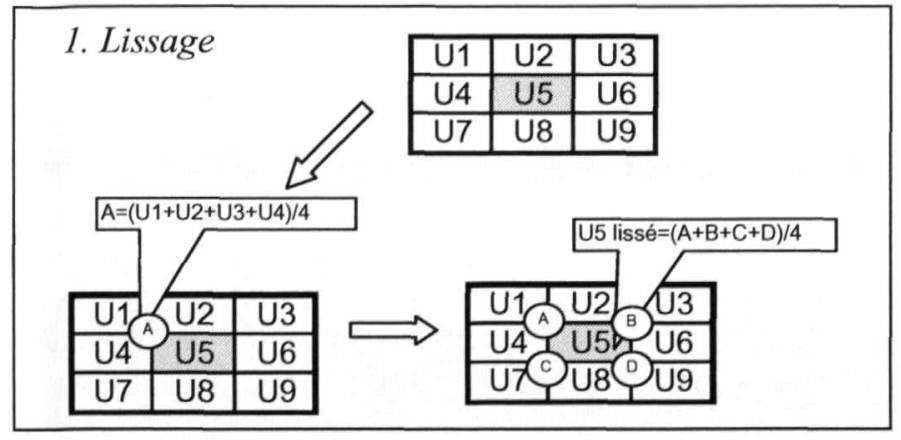

\section{Echantillonnage}

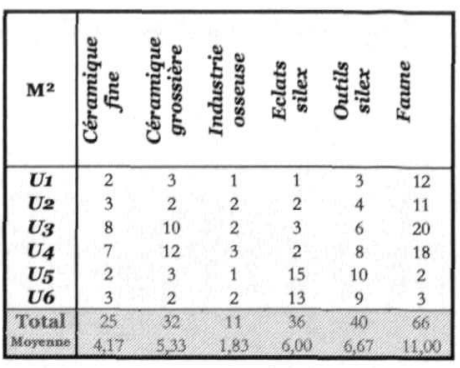

Tableau de données

\section{Analyse Factorielle}

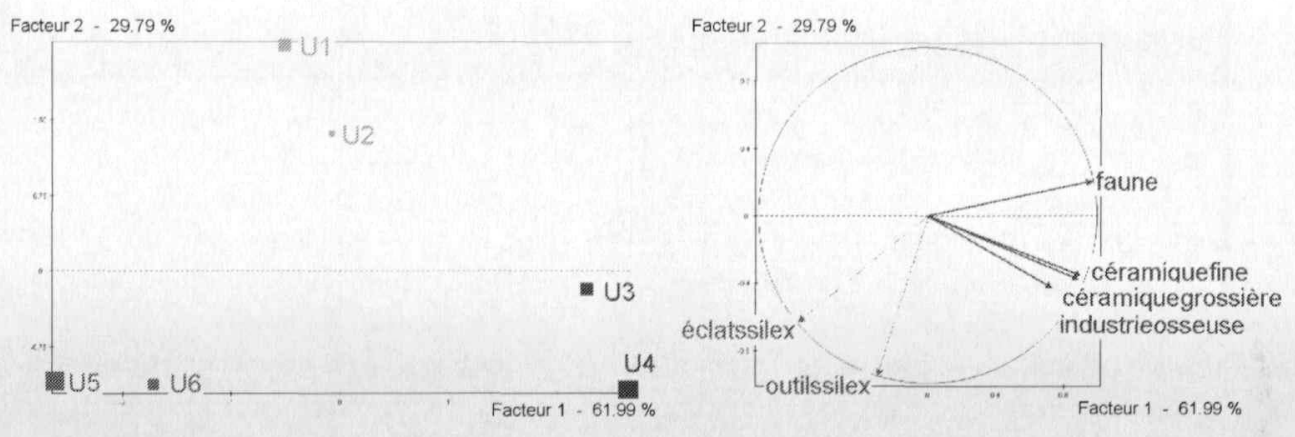

\section{Classification}

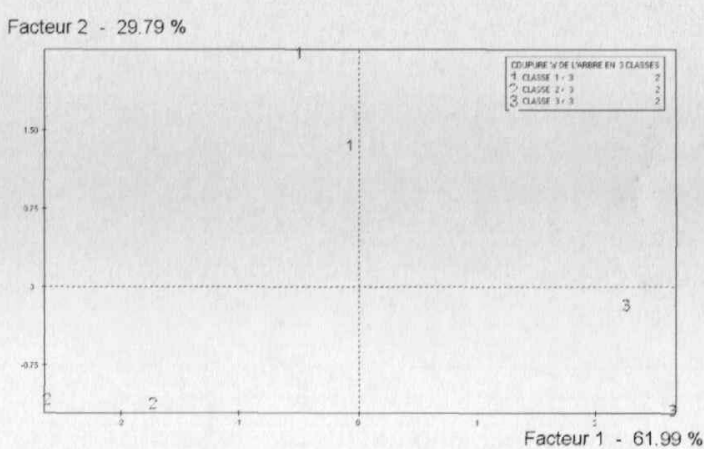

\section{Caractérisation}

\begin{tabular}{|c|c|c|}
\hline Classe 1 & Classe 2 & Classe 3 \\
\hline Irés peu de vestiges & Riche en silex : éclats et outils & $\begin{array}{l}\text { Riche en céramique fine et } \\
\text { grossière et en faune }\end{array}$ \\
\hline Unites $1: 2$ & Unités $5 \& 6$ & Unités $3 \& 4$ \\
\hline
\end{tabular}

\section{Interprétation}

Figure 1 : Schématisation de la méthode de « structuration spatiale ».

Figure 1: A short summary of the method used in spatial analysis presented in the text.

\section{Echantillonnage}

Il est échantillonné des vecteurs d'effectifs. Nous avons choisi d'utiliser la division du carroyage.

\section{Analyses factorielles}

Des analyses factorielles sont réalisées afin de mettre en évidence des facteurs d'association/répulsion des catégories de vestiges. Elles permettent aussi 
de diminuer les facteurs parasites en les ôtant, ou en supprimant leur poids analytique en les rendant illustratifs (cas des modalités à trop faible ou trop forte contribution ou à trop faible effectif).

L'analyse factorielle permet d'étudier l'évolution des variables dans l'espace, c'est-à-dire la distribution de leurs différentes modalités sur les graphiques. Ainsi, nous pouvons mieux comprendre leurs liens avec les unités de fouilles. Un atout de la méthode est de procurer des résultats autant sur les variables que sur les individus. Ainsi la question se pose en termes de relations entre les vestiges les uns par rapport aux autres et de comportement des unités de fouille par rapport aux vestiges.

\section{Classification}

Les analyses factorielles réalisées dégagent des tendances de regroupement ou d'opposition entre les vestiges, entre les unités de fouilles et entre les unes et les autres. Les méthodes de classification ont pour objectif de faire apparaître des groupements dans un ensemble de données par partition, les individus ou les variables mais pas les deux. "Ces groupements sont progressifs, hiérarchisés et sont effectués de manière progressive par agglomération (classification ascendante) ou dégressive (classification descendante) 》 (Demoule et al., 2002).

Dans le cas d'une classification ascendante hiérarchique (Cah), les objets sont pris individuellement et comparés entre eux à l'aide d'une mesure de proximité ou de ressemblance, puis sont regroupés pas à pas jusqu'à former un seul ensemble. L'inverse est le cas d'une classification descendante (Cdh) : on part d'un ensemble global pour le subdiviser. Les classes sont visualisées sous la forme d'un arbre de classification ou dendrogramme. Celui-ci peut ensuite être découpé à plusieurs niveaux, selon sa morphologie et le nombre de classes souhaitées. Ainsi, à partir d'une classification, on peut définir avec finesse les degrés de ressemblance en les quantifiant et, surtout, une classification des unités de fouille en fonction de leur degré d'association et/ou répulsion de vestiges repérés. Cela permet des rapprochements fins et chiffrés.

Par ailleurs notons que le nombre de classes influe sur l'échelle d'observation : avec un nombre de classes faible, on analyse les phénomènes à une échelle globale ; avec un plus grand nombre de classes on va vers une échelle de traits spécifiques plus fine.

\section{Caractérisation des classes}

Le contenu des classes est détaillé puis, les unités de fouilles identifiées par leur classe d'appartenance sont reportées sur un plan afin d'en connaître leur extension horizontale. Cette caractérisation permet de voir si les associations de vestiges sont continues, récurrentes d'un espace à l'autre, de tailles comparables...

\section{Interprétation}

Une interprétation peut alors être proposée à partir des résultats obtenus sur ces organisations spatiales de vestiges. Que signifient les associations des vestiges repérés? Pour les interpréter il est commode de faire appel à des analogies. Ces analogies sont issues, tantôt du monde contemporain (modèles ethnologiques), tantôt de con- naissances développées antérieurement ou décrites par d'autres chercheurs (modèles archéologiques)...

Existe-t'il des zones issues de rejets spécifiques ou multiples, primaires ou secondaires? Existe-t'il des zones nettement délimitées dans l'espace (des aires) qui soient le siège d'activité spécifiques, des aires de rejet, des aires de combustions ... ? Qu'y était-il pratiqué : des activités artisanales ? des tâches domestiques (tâches à effectuer quotidiennement au sein de la maisonnée telle que la préparation des aliments)?

Les activités pratiquées, reflétées par les restes au sol, étaient-elles complémentaires, autonomes, opposées dans leur production, à utilisation spécifique?

\section{Application archéologique}

\section{A. Egolzwil 3}

Le village d'Egolzwil 3 situé dans le Wauwilermoos (Lucerne, Suisse) s' intègre au plus large contexte des stations du marais de Wauwil (Egolzwil 1-2-4 et 5) au centre du plateau Suisse. La fouille de la station 3 débute dans les années 1950 en raison de la dégradation rapide du site. La couche archéologique s'étend de 496.50 à 498.00 mètres d'altitude, mais la conservation des vestiges organiques est absente au delà de 497.00 mètres. En effet, entre les premiers sondages de A. Greber et A. Graf en 1933, et la fouille de 1985, il s'est opéré un tassement des couches supérieures. Les sondages de 1933 montraient au dessus du foyer $1 / 1985$ (dans la zone est), $110 \mathrm{~cm}$ de couche puis, en 1950 , seulement $100 \mathrm{~cm}$ et enfin, en 1985, seul $90 \mathrm{~cm}$ étaient encore présents. Ces processus post-dépositionnels n'ont toutefois pas trop affecté les niveaux archéologiques; seules les chapes d'argiles de la partie supérieure de la zone centrale du village située du côté du lac sont affectées par l'érosion et ce, uniquement dans leurs parties supérieures.

Les opérations de fouille ont débuté durant les années 1950-1952, sous la direction d'E. Vogt, dans la partie centrale qui, selon le système actuel de coordonnées, va de A82 à A 100. Les fouilles furent ensuite reprises de 1985 à 1988 sous la direction de R. Wyss et publiées sous forme de monographie en 1996. Au total une surface de $1800 \mathrm{~m}^{2}$ environ est ainsi décapée. Les techniques de fouille ont évolué entre ces deux campagnes nous amenant à ne prendre en compte que les opérations de fouilles les plus récentes, donc en ôtant les surfaces dégagées par E. Vogt (bandes représentées en gris).

Tous les vestiges, dont les pieux repérés, sont relevés en coordonnées $\mathrm{X}, \mathrm{Y}, \mathrm{Z}$. La délimitation du plan au sol des maisons est faite sur la base de l'emplacement des chapes d'argile comportant des signes d'exposition au feu (traces rouge-noir, particules de charbon, zones rubéfiées, couches de cendres), chapes ayant été interprétées comme des structures foyères. En effet, d'autres plaques sont relevées mais ne comportant aucune trace de rubéfaction. Les pieux classés en fonction de la détermination des espèces ont parallèlement été étudiés. Les constructions sont réalisées à $78 \%$ avec du frêne, du chêne et de l'aulne puis, à $13 \%$ avec des saules, de l'érable et du peuplier. Enfin, le reste est constitué de $9 \%$ avec le noi- 
setier, l'orme, le tilleul et le bouleau. De façon plus anecdotique, on trouve du sapin blanc. Ce sont dans les zones nord ouest et ouest que les plans sont apparus le plus clairement. Au total, il a ainsi été décelé 24 structures architecturales posées à même le sol dont 2 incomplètes. La dimension moyenne des bâtiments est de 8,5 mètres de long sur 4 mètres de large. Le village est entouré d'une palissade du côté du lac.

Après plusieurs épisodes d'inondations durant l'occupation, le village est définitivement abandonné, laissant les hommes emporter les outils encore utilisables n'offrant donc aux archéologues que les objets usagés ou les rejets ! Les débuts de la station d'Egolzwil 3 sont datés par radiocarbone (Institut physique de l'université de Berne ath-131) de 4282 - 4280 BP.

\section{Analyses préliminaires}

\section{$>\quad$ Données et variables retenues}

Les vestiges relevés lors de la fouilles concernent tant les vestiges minéraux ou fossilisés : industries osseuses ou lithiques (silex, roches dures, gros lithique...), la faune, et la céramique, que les vestiges organiques : bois, vannerie, sparterie... Les sept catégories de vestiges retenues sont décomptées (comptage, mesure ou pesage) en fonction de leur nature ${ }^{2}$. D'autres catégories de vestiges sont écartées car elles ne reflètent pas des activités menées sur place à l'image de l'outillage poli.

Céramique. Les tessons de céramique fine ou grossière sont pesés en grammes et ne sont pas ici différenciés, la très faible représentation de la céramique fine ne pouvant constituer seule une catégorie statistiquement représentative.

Industrie lithique. L'industrie lithique se divise entre les éléments relevant du débitage avec les éclats (comportant ou non des retouches volontaires) et les produits finis, outils brisés ou entiers (grattoirs, couteaux, burin, perçoirs, pointes, pointes de flèches...). Les pièces sont pesées en grammes, avec une unité minimale d'un gramme.

Industrie osseuse. L'industrie osseuse ne comporte que des outils finis, brisés ou entiers : poinçons, pointes, ciseaux, spatules, aucune ébauche n'est présente. Cette catégorie est comptée et pesée (le poids exprimé en grammes).

Vestiges fauniques. Ils concernent tous les restes fauniques, déterminés ou non, toutes espèces confondues domestiques et sauvages. Le système de comptage employé est le dénombrement. Nous aurions souhaité procéder à un pesage systématique mais cela ne nous a pas été possible.

Outillage de mouture. Il comprend les meules et les molettes sans différence typo-technologique. Elles sont mesurées (longueur, largeur, épaisseur) et pesées (le poids exprimé en grammes).

Percuteurs. Ce sont tous les outillages de pierre comportant au minimum une surface de percussion. Le dénombrement est réalisé selon les dimensions et les poids (le poids exprimé en grammes).

Une fois la base de données établie, les données sont lissées à partir des huit carrés adjacents. Le tableau de données servant de base à notre analyse est donc un tableau de données mètres carrés / catégories de vestiges avec à l'intersection les quantités lissées (tab. 2).

\section{Plans de répartition}

Afin d'appréhender le fonctionnement de chaque catégorie de vestiges, les distributions des données brutes et lissées sont mises en plans. Les quantités présentes dans chaque mètre carré sont représentées par des symboles proportionnels aux effectifs. Les classes des symboles sont établis à partir du découpage de l'histogramme des quantités par mètres carrés. Cette représentation synthétique fait ressortir visuellement les structures de densité et permet ainsi déjà de repérer les zones homogènes ( $c f$. fig. 2).

Les plans sont décrits par groupes en raison de similarités formelles. En effet, les plans de répartitions forment deux ensembles. Le premier se constitue des vestiges se répartissant d'une façon homogène à travers le village. Ce sont l'industrie osseuse (pointes, poinçons, ciseaux, spatules), la faune (caprinés, suidés et cervidés essentiellement), les percuteurs et les outils de mouture (meules et molettes). A l'inverse, le second groupe montre des oppositions entre les zones Est et Ouest du village ; il est formé par les tessons de la céramique et les éclats lithiques.

En rapportant ces distributions avec les plans de maisons établis par la dendrochronologie et les chapes d'argiles, il ressort une nette dichotomie entre les zones intérieures et extérieures des unités d'habitation. En effet, circonscrits à la périphérie des chapes d'argile, se trouvent des outils en os et des restes fauniques. Les meules et les molettes se retrouvent également à l'intérieur, mais légèrement excentrées par rapport à la zone foyère. Les

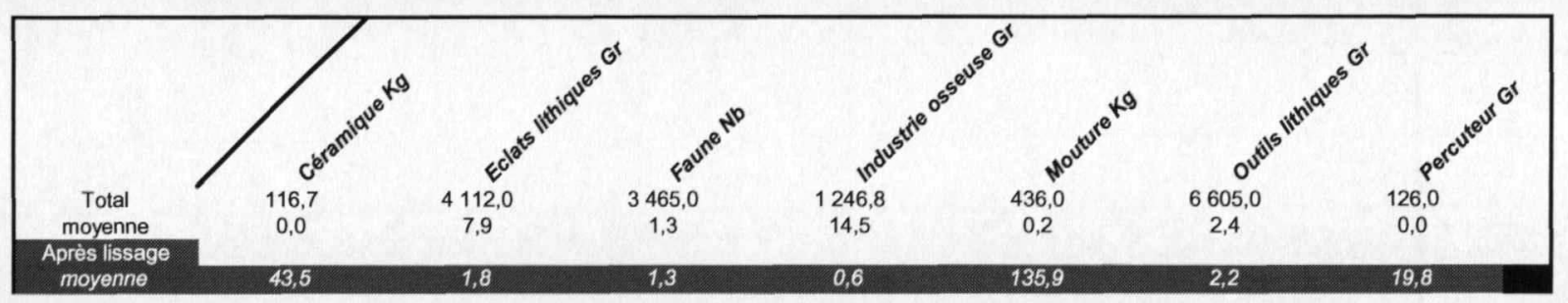

Tableau 2 : Résumés statistiques des catégories de témoins du site d'Egolzwil 3.

Table 2: Statistical summary of remains categories of the site of Egolzwil 3.

\footnotetext{
${ }^{2}$ Cet inventaire nous a été rendu possible grâce à M. Wyss, Fasnacht et Nielsen qui m'ont permis d'accéder aux collections avec une grande facilité.
} 

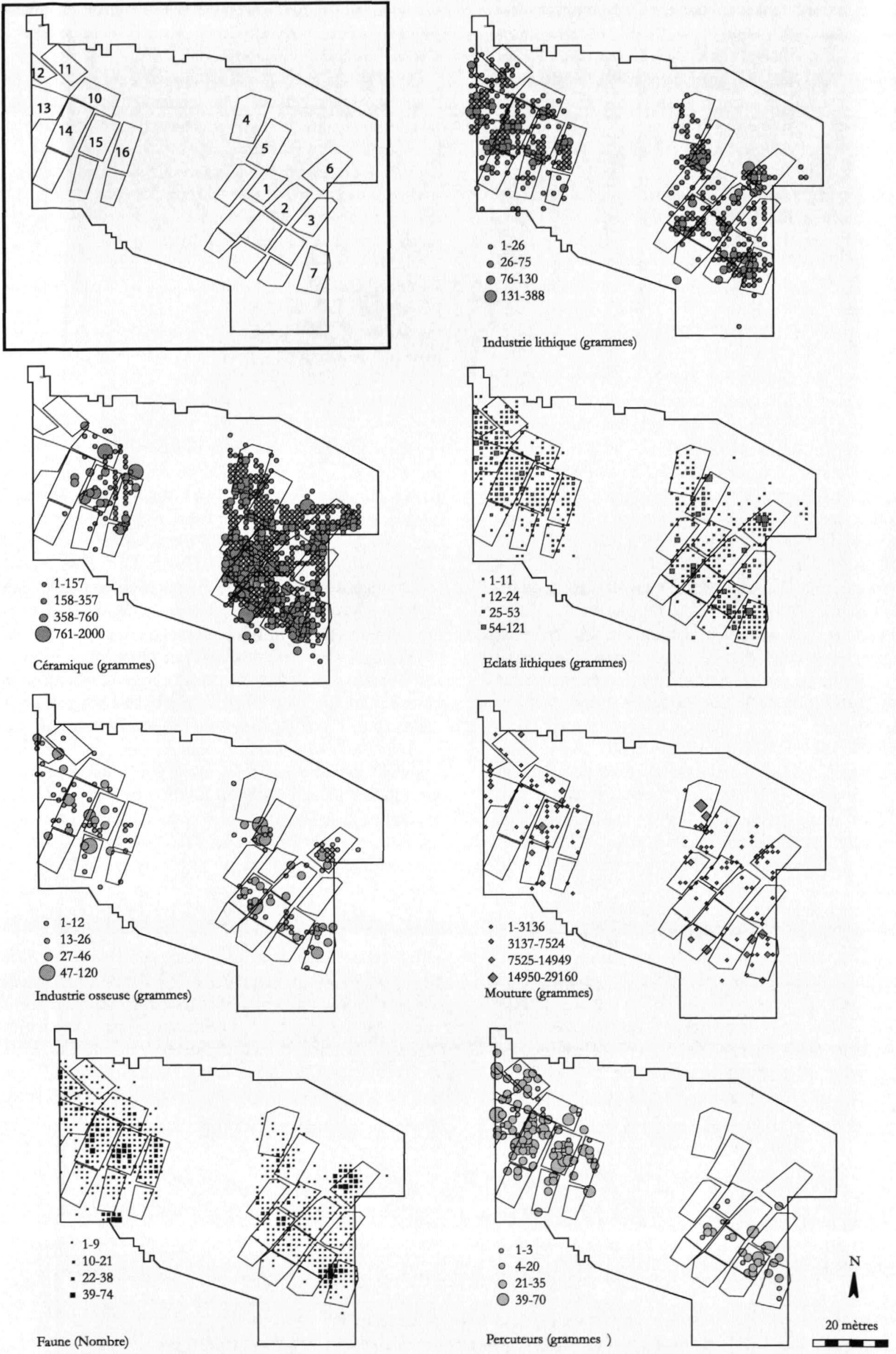

Figure 2 : Plan de répartition des témoins sur la station d'Egolzwil 3.

Figure 2: Horizontal distributions of archaeological remains of the site of Egolzwil 3. 
nappes ont tendance à s'étaler vers l'extérieur, comme si ces activités se déroulaient toujours sur la limite intérieure/extérieure, ou à proximité d'une ouverture. Enfin, les percuteurs, dont les concentrations sont nettement délimitées se retrouvent quasi exclusivement au sein des unités domestiques.

Quelles sont exactement les différences entre les zones Est et Ouest? Quels sont les rapports avec les structures architecturales ? Toutes les structures sont-elles de même nature?

\section{Analyses factorielles}

Plus que de saisir les relations entre les variables observées à l'aide des plans de répartition, nous voulions comprendre par le biais des constitutions de vestiges associés, les liens que les unités de fouilles entretenaient les unes avec les autres ( $c f$. fig. 3). Ainsi, une analyse en composantes principales (ACP) a été réalisée sur le tableau de données mètres carrés / catégories de vestiges avec à l'intersection les quantités lissées.

\section{Présentation des résultats chiffrés}

- Pourcentage d'inertie sur les quatre premiers axes factoriels

L'histogramme des pourcentages d'inertie permettant de vérifier la validité statistique de l'analyse est élevé sur le premier axe $(37,8 \%)$ pour chuter rapidement, n'étant plus sur le second axe qu'à (14,6\%). Sur les deux axes suivants (3 \& 4), le pourcentage d'inertie atteint (13,7\%), puis $(11,7 \%)$, pour ensuite ne quasiment plus décroître que de quelques pourcents sur les axes suivants.

Les pourcentages cumulés sur les quatre premiers axes donnent plus de $78 \%$, ce qui semble, d'une part satisfaisant pour une telle analyse et, d'autre part, suffisant pour interpréter les résultats fournis par l'analyse, puisque les axes suivants ont un contenu informatif faible, allant vers des spécificités difficiles à appréhender. On peut dire que les résultats précédemment décrits reflètent, du point de vue mathématique, une analyse significative autorisant une exploitation.

\section{- Pourcentage de corrélation des variables}

Seules seront présentées les corrélations élevées, représentatives de faits archéologiques, repérées depuis la matrice des corrélations qui présente les pourcentages de corrélation entre les répartitions de vestiges.

D'emblée, nous pouvons noter qu'il n'existe pas de corrélations linéaires négatives, c'est-à-dire qu'aucune distribution de vestiges ne s'exclut. Trois corrélations élevées existent entre les éclats de silex et la faune (0.61), les éclats et les outils de silex (0.50), et enfin, les outils de silex et la faune (0.48). Ces taux assez élevés rejoignent les observations des plans de répartition, pour montrer les organisations similaires de ces vestiges. Viennent ensuite des corrélations moins importantes aux alentours de $(0.35)$; elles existent, par ordre décroissant, entre la faune et l'industrie osseuse, les outils en silex et l'industrie osseuse, les outils en silex et la mouture, la céramique et la faune, la céramique et les éclats de silex. $>\quad$ Présentation des résultats graphiques des analyses factorielles

- Plans factoriels

Organisation des variables

La description des plans factoriels se fait en liant les axes deux à deux selon leur ordre de pourcentage d'inertie: ces liaisons nous apparaissent les plus explicites pour la compréhension de l'analyse. Superposer graphiquement les plans des variables et des individus étant impossible dans le cadre d'une analyse en composantes principales, les plans sont examinés parallèlement.

\section{Sur le plan factoriel axes 1 - axe 2}

Les individus s'organisent en triangle, avec, à deux extrémités, l'outillage de mouture et les percuteurs qui se démarquent en positif sur les deux axes, tandis que le second groupe, constitué des éclats et des outils en silex, de l'industrie osseuse, de la céramique et de la faune, est en positif sur l'axe 1 mais en négatif sur l'axe 2. Des groupes d'individus semblent également nettement se former autour de ces groupes de vestiges. Notons, tout de même, que la majorité des individus sont réunis à la pointe du triangle du côté de l'origine des axes.

\section{Sur le plan factoriel axes 3 - axe 4}

Les variables sont aussi dispersées sur les axes (3-4) que sur les axes précédents. Les mètres carrés exempts de vestiges se trouvent toujours sur l'origine des axes mais, cette fois, en association avec les unités de fouille dans lesquelles ne sont représentés que les vestiges fauniques ou dans lesquelles ne sont présents que des éclats de silex.

Par ailleurs, quatre groupes se démarquent nettement, tant au niveau des variables que des individus. Ce sont les percuteurs qui s'isolent en négatif sur les deux axes, la céramique, l'outillage de mouture ; le dernier groupe se constitue de l'industrie osseuse et lithique. Ces groupes se forment, tant par la spécificité des mètres carrés qui les composent que, en opposition les uns par rapport aux autres. En effet, peu d'individus s'isolent d'un groupe.

\section{Découpage en classes automatiques}

Une classification ascendante hiérarchique (CAH) est effectuée sur les coordonnées des mètres carrés présents sur les axes factoriels. Afin d'éviter une description longue et fastidieuse, nous ne citons que les partitions de l'arbre de classification conservant du sens sans les décrire finement. En effet, un tel développement serait récurrent avec la description de la partition en neuf classes choisie pour la compréhension de la structuration du village d'Egolzwil 3. Il est possible de couper en 3, 6 et 9 classes. Toutefois, une partition en trois classes ne fourni qu'une faible quantité d'informations en raison $\mathrm{du}$ manque de précision. Dans une partition en six classes, les grandes classes sont les mêmes que dans le découpage finalement employé mais la précision est moindre. L'avantage du découpage en 9 classes est de conserver des phénomènes de groupes tout en évitant une masse de cas particuliers. 


\section{Projection des résultats sur les plans factoriels}

Les axes décrits dans les paragraphes qui suivent sont au nombre de 4 , résumant plus de $78 \%$ de l'information (tab. 3).

\section{Sur le plan factoriel axes 1 - axe 2}

La structure du graphique des axes (1-2) est la même que dans la description de l'analyse factorielle. Les classes se répartissent autour de la structure triangulaire. Ainsi, les individus s'organisent en triangles avec la classe 1 représentée sur l'origine des axes. Ce sont en

\section{Analyse en composantes principales}
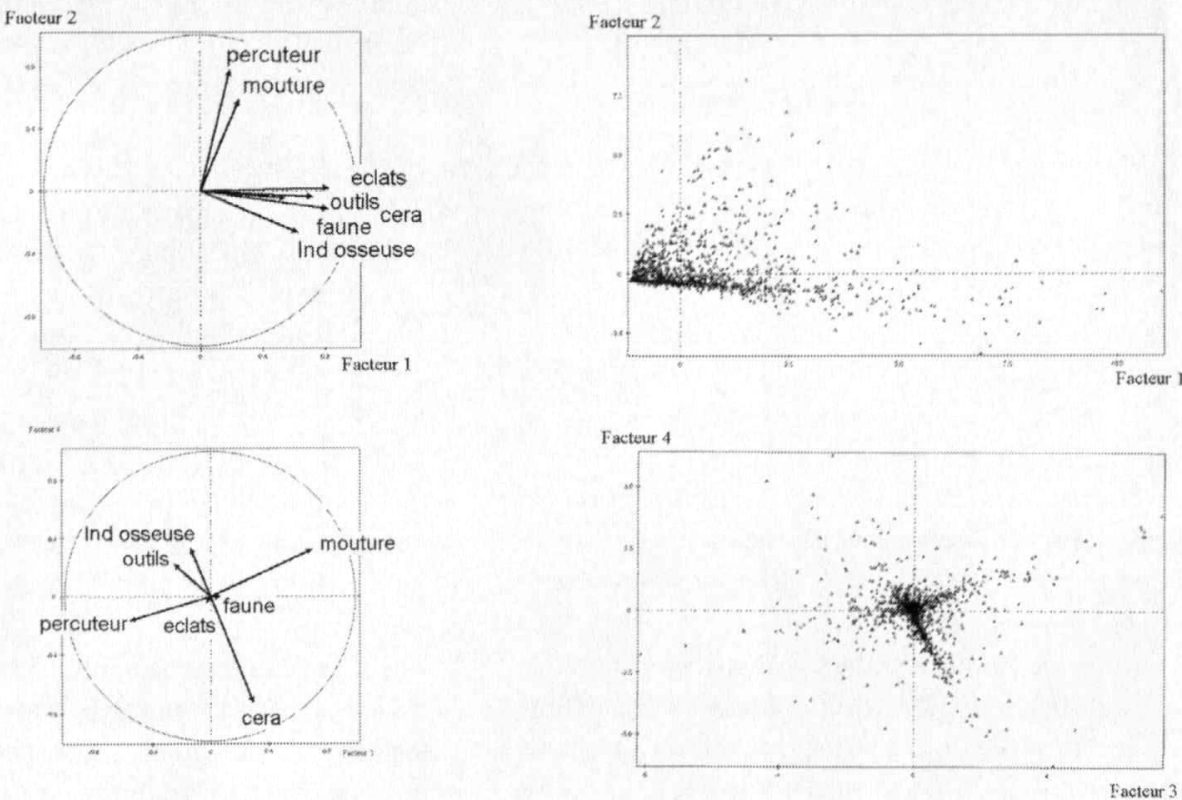

\section{Classification ascendante hiérarchique}
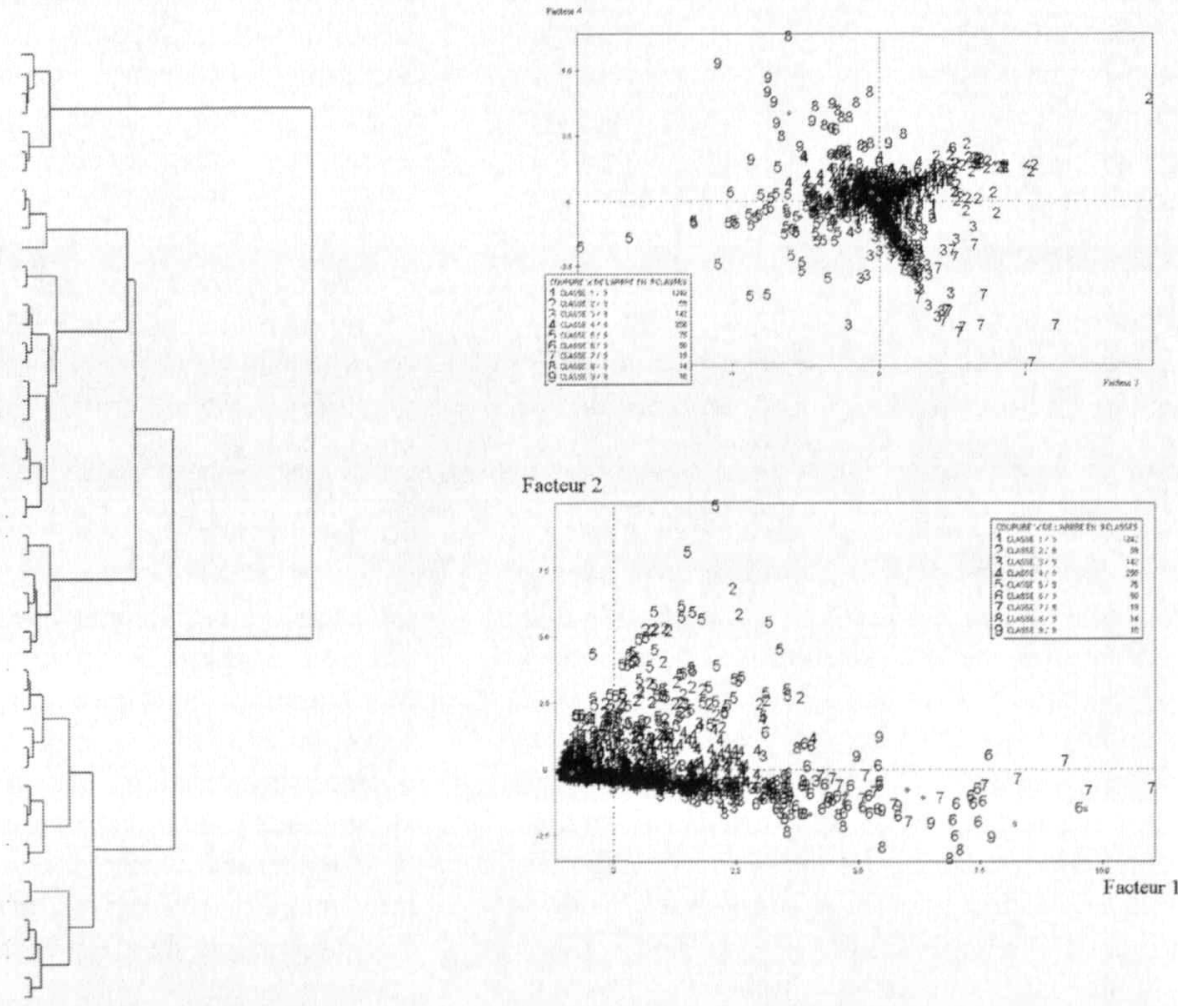

Figure 3 : Résultats graphiques de l'analyse en composantes principales d'Egolzwil 3.

Figure 3: Graphical results solved by multidimensional methods of the site of Egolzwil 3. 


\begin{tabular}{|c|c|c|c|c|c|c|c|c|c|}
\hline Glasse & $I$ & 2 & 3 & 4 & 5 & 6 & 7 & 8 & 9 \\
\hline $\begin{array}{c}\text { Vestiges } \\
\text { principaltx }\end{array}$ & $\begin{array}{l}\text { Fixcmpts dc } \\
\text { vestiges } \\
\text { particulicrs }\end{array}$ & Mouturc & Córamique & $\begin{array}{l}\text { Ficlats (Jutils } \\
\text { faune }\end{array}$ & Percuteurs & $\begin{array}{c}\text { Iaune I.clats } \\
\text { silex Outils } \\
\text { silex Industrie } \\
\text { osscusc }\end{array}$ & $\begin{array}{l}\text { Ceramique } \\
\text { liaune }\end{array}$ & $\begin{array}{c}\text { Industrie } \\
\text { osseuse faune }\end{array}$ & $\begin{array}{l}\text { Outils silcx } \\
\text { Industrie } \\
\text { osseuse }\end{array}$ \\
\hline $\begin{array}{c}\text { Vestiges } \\
\text { secondaires }\end{array}$ & & & $\begin{array}{c}\text { Industrie } \\
\text { osscusc Fraune } \\
\text { Fclats silex }\end{array}$ & & $\begin{array}{l}\text { Outils silex } \\
\text { Fclats silex }\end{array}$ & Céramique & $\begin{array}{c}\text { Fclats silex } \\
\text { Outils silex } \\
\text { Industrie } \\
\text { osscusc } \\
\text { Mouture }\end{array}$ & Felats silc $x$ & $\begin{array}{c}\text { Fclats silex } \\
\text { Faune }\end{array}$ \\
\hline
\end{tabular}

Tableau 3 : Composition des classes automatiques issues de la classification ascendante hiérarchique d'Egolzwil 3. Table 3: Final results of multidimensional methods authorizing us to group remains categories of Egolzwil 3.

effet, tous les mètres carrés de fouille ne comportant que peu ou pas de vestiges qui se réunissent au sein de cette classe. Les autres individus sont plus éclatés formant tout de même des groupes assez nets autour de leurs classes d'appartenance.

\section{Sur le plan factoriel axes 3 - axe 4}

Les informations précisées par les axes 3 et 4 sont assez similaires à celles apportées par les deux axes précédents. Ils exacerbent toutefois les caractéristiques particulières aux dépens de la réunion, au centre sur l'origine des axes, des classes 1,4 et 6 les plus représentées.

\section{Interprétation spatiale des classes}

\section{Interprétation de chaque classe en fonction de sa nature et de sa localisation}

Chaque classe se représente sur un minimum d'une vingtaine de mètres carrés. Cette caractéristique est notoire car elle tend à montrer que les vestiges ont peu bougé puisque l'on observe une constante dans les débris liés les uns avec les autres dans des espaces topographiquement différents. Ainsi, loin de s'individualiser en un endroit unique et en une grande étendue, chaque classe se répète à plusieurs reprises dans le village. Ce sont ces récurrences qui autorisent à saisir la structuration du village. Par ailleurs, ces surfaces récurrentes par classes montrent une relation tant avec les structures foyères qu'avec les structures architecturales identifiées par $R$. Wyss. Autant d'indices supplémentaires qui nous permettent d'affiner notre interprétation de l'espace de la station.

La classe 1 ne connaît que des valeurs négatives sur l'axe factoriel 1 , les vestiges qui la composent étant en quantité inférieure à leur moyenne sur le site. Ainsi, la classe 1 permet de repérer les zones sur lesquelles aucune activité n'abandonnant des vestiges en grande quantité n'est pratiquée. Ces espaces, majoritaires sur la surface, représentent $1242 \mathrm{~m}^{2}$ sur un total de $1869 \mathrm{~m}^{2}$ étudiés.

La classe 2 est exclusivement composée du matériel de mouture (meules et molettes). Elle se retrouve en majorité dans les structures, sans toutefois être omniprésente (elle est absente des maisons $2 \mathrm{a}$ et $2 \mathrm{~b}$, la et $1 \mathrm{~b}, 15 \mathrm{a}$ et $15 b$ et $16 \mathrm{a})$. La localisation des meules et des molettes, à l'exclusion de tout autre type de restes, n'est pas stan- dardisée. Les outils de mouture se retrouvent rarement autour du foyer (15a), mais le plus souvent sont repoussés vers les parois latérales ou longitudinales des habitations. Cette localisation doit répondre à une nécessité fonctionnelle de ranger ces outils conséquents le long des parois ou d'évacuer les rejets assez encombrants comme ceux occasionnés par l'exécution de certaines activités.

La classe 3 se caractérise par une très forte proportion de céramique, associée à quelques éclats de silex et outils en os. Cette classe se retrouve quasi exclusivement dans la partie Est du village, soit dans les maisons la et $1 \mathrm{~b}, 2 \mathrm{a}$ et $2 \mathrm{~b}, 3 \mathrm{a}$ et $3 \mathrm{~b}$ et 7 et à l'Ouest dans la maison $15 \mathrm{~b}$.

La classe 4 , localisée aux alentours des foyers, est constituée d'éclats et d'outils en silex, de restes fauniques et de quelques outils en os. Ces indices semblent montrer des activités de subsistance directe telles que les préparations culinaires (cuisson, préparation des repas...).

La classe 5, constituée de percuteurs, d'éclats et d'outils en silex, est présente dans les structures principalement de l'Ouest du village $13,14 a$ et $b, 15 a$ et $b, 16 a$ et $b$ et dans la partie Est, seulement dans les structures 5 et 6 . Ces zones, très localisées et spécifiques doivent pouvoir être mises en relation avec les zones de production faisant appel à cet outillage (taille).

La classe 6, composée de restes fauniques, d'éclats et d'outils de silex, d'industrie osseuse et d'un peu de céramique est présente au niveau des foyers uniquement dans quelques maisons. Son interprétation est à rapprocher de celle de la classe 4 , en raison de la nature de ses vestiges. Toutefois, il est à y noter l'importance des outils et des éclats en silex.

La classe 7, formée essentiellement de céramique et de faune, avec quelques outils et éclats de silex se retrouve au niveau des foyers dans les structures $3 \mathrm{~b}$ et 7 . La signification de cette classe est à rapprocher de celle de la classe 3 . Son existence provient certainement d'un mélange produit lors de l'occupation.

La classe 8 est constituée d'une très forte majorité d'outillages en os, associés à quelques éclats et restes fauniques. Cette classe matérialise les espaces d'utilisation de ce type d'outillage. En effet, elle ne comporte ni fortes quantités absolues ni catégories typologiques spécifiques, ce qui ne permet pas de trancher catégoriquement pour des lieux de production spécialisés. Toutefois, des différences avec l'outillage de base existant dans chaque 
maison sont très nettes. Ces espaces se retrouvent au sein des maisons 15b, 5 et 6 (les plans de répartition montrent également une zone assez riche dans la structure 1b).

La classe 9 se répartit principalement dans les structures de l'Ouest du village $13,14 \mathrm{a}$ et b, 15a et b, 16a et $b$ et dans la partie Est dans les structures 5 et 6 . Ces nappes, assez denses, se constituent majoritairement d'outils en silex. Comme pour les deux classes riches en tessons céramiques, la classe 9 est assez peu représentée. En effet, de nombreux outils et éclats en silex issus de la classe 4 , et destinés aux usages quotidiens, existent au sein de chaque maisonnée. Ainsi, la classe 9 ne montre que les très fortes concentrations de silex. Ces espaces, peut-être liés à la taille, ne présentent pas de localisation préférentielle au sein des structures, mais il est tout de même à noter que, majoritairement, ces zones se situent à proximité des parois.

Plan du site avec répartition des classes

L'élément qui ressort de l'examen du plan est donc la grande étendue des nappes ( $c f$. fig. 4 \& 5).
Le premier élément se démarquant est le contraste existant entre les espaces exempts de vestiges, individualisés par la classe 1 , et les autres espaces quelle que soit leur constitution en vestiges. Cette différence reflète l'opposition entre les zones intérieures et extérieures, rejoignant dans les grandes lignes le plan des structures suggérées par Wyss. On retrouve les maisons alignées en une rangée parallèle au lac.

Un certain nombre de critères permettent de reconnaître les structures architecturales comme des unités d'habitations; ainsi, les classes 2 et 4 (dont la composition des aires d'activités) s'y retrouvent de façon récurrente. Leurs vestiges sont issus des activités nécessairement quotidiennes telles que la subsistance directe (cuisson, préparation, consommation des repas...). Ces activités se déroulent naturellement près de la zone foyère. De même, la classe 6 , comporte une plus forte proportion d'outils en silex. Sa formation, à mettre en rapport avec la constitution de ses classes voisines, nous amène à nous interroger sur les autres activités pratiquées dans les espaces intérieurs.

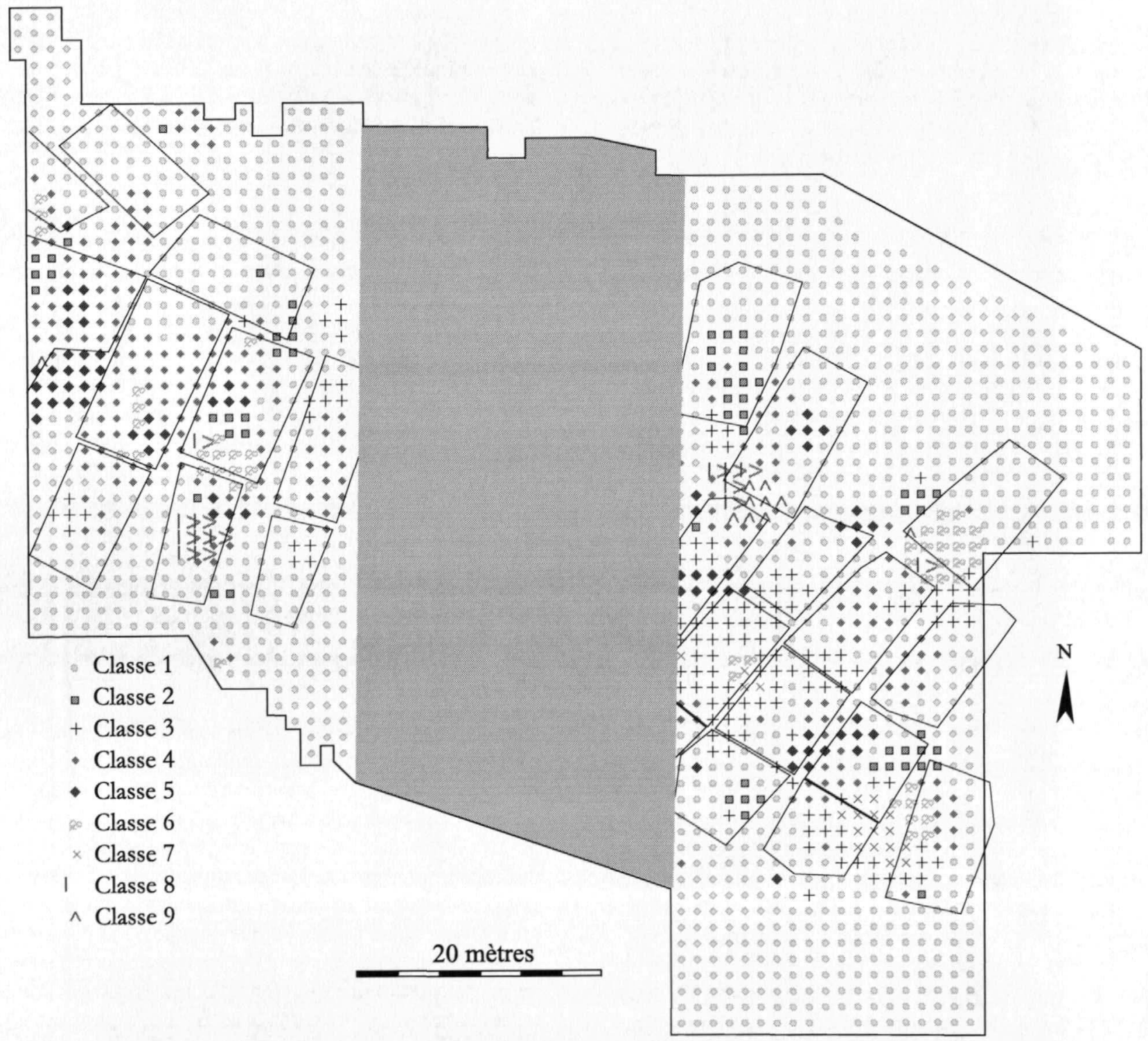

Figure 4 : Découpage en classes reporté en plan sur la station d'Egolzwil 3.

Figure 4: Statistical results reported in a map on the site of Egolzwil 3. 


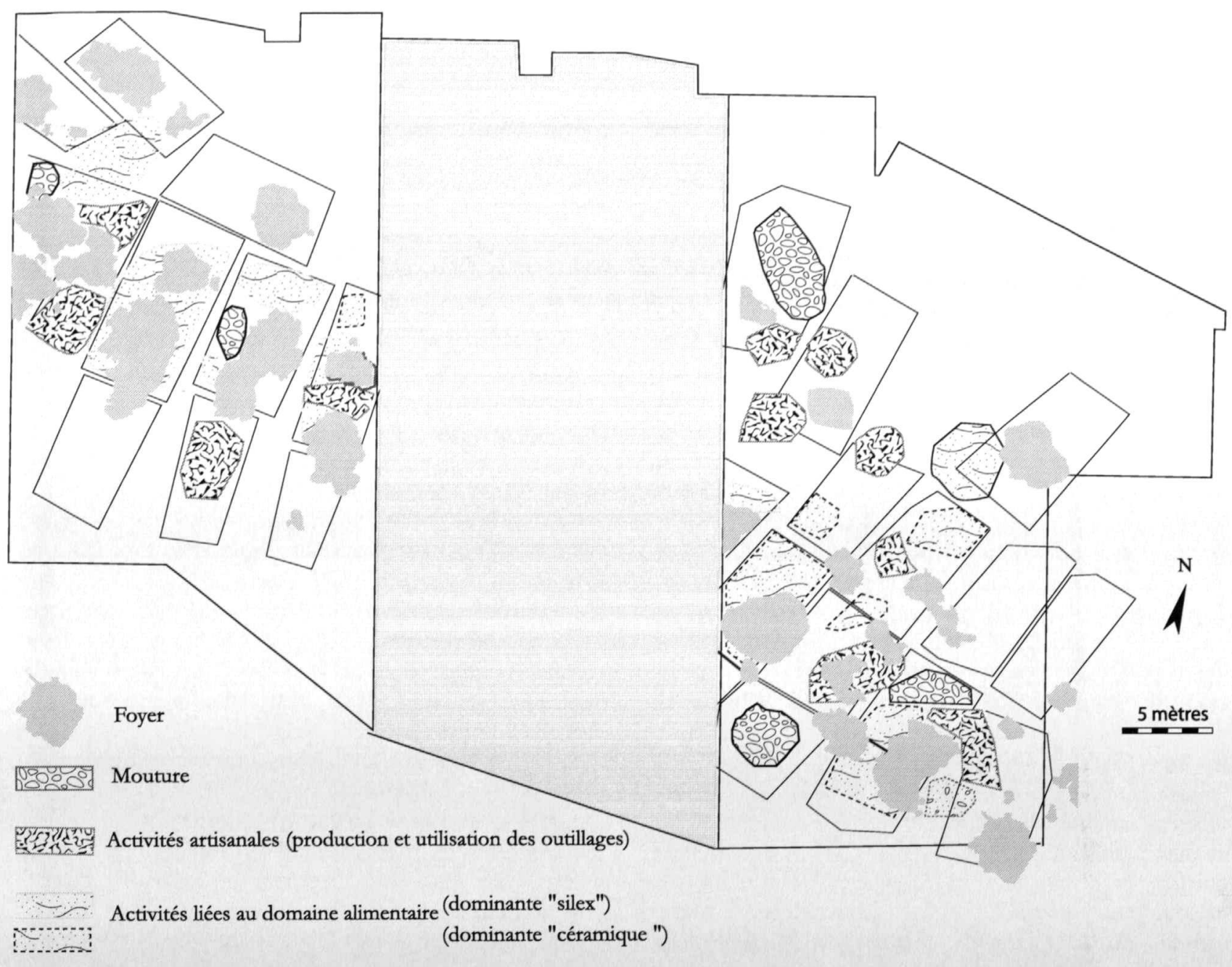

Figure 5: Bilan de l'organisation spatiale de la station d'Egolzwil 3.

Figure 5: Syntheses of the spatial organisation of the site of Egolzwil 3.

Il ressort, alors, un comportement dichotomique des vestiges, comportement déjà repéré dans les plans de répartition décrits plus haut. En effet, le village semble se diviser en deux zones : les groupes de maisons plus riches en céramique, avec la classe 3 , et celles plus riches en restes de taille de silex, avec la classe 5 . Il serait nécessaire de compléter ces approches avec une étude orientée vers la reconnaissance de la nature des activités pratiquées dans chaque maison et d'éventuelles productions spécialisées. Cette seconde étape analytique requiert alors des informations qualitatives plus précises. Une telle étude a été entreprise par R. Wyss (1996) qui, à partir de répartitions de vestiges par catégories typologiques, montre des spécificités dans certaines maisons. Ainsi, la structure 14 $(\mathrm{a} \& \mathrm{~b})$ particulièrement riche en haches de combat et contenant, en os, la structure 15 (a \& b) de harpons et la structure 5 vue comme un lieu de fabrication de haches.

\section{B. Charavines-les-Baigneurs}

Le site des Baigneurs se situe sur les rives du lac de Paladru à 495 mètres d'altitude dans les Alpes septentrionales. Ce lac à beine est occupé à deux reprises durant le Néolithique final. La station a été fouillée sur 450 mètres carrés sous la direction d'A. Bocquet durant 14 ans (de
1972 à 1986). Depuis la fin des fouilles, une grande partie du matériel recueilli a été étudié nous permettant de nous inscrire dans un contexte typo-techno culturel fin. La première occupation, datée par dendrochronologie, pour les poteaux les plus anciens, de 2669 av. J.-C., sur un sol vierge dura une vingtaine d'années. Le village est ensuite abandonné une quarantaine d'années, laissant une épaisse couche stérile qui sépare clairement les deux occupations. L'abandon progressif du village pour le déplacer (Bocquet, 1994) après avoir épuisé les terres alentours auraient d'une part, laissé le temps aux habitants d'emporter avec eux les outils encore utilisables et d'autre part, permis aux vestiges organiques laissés à l'air libre de se décomposer en partie. La couche se retrouve donc principalement constituée d'éléments minéraux.

Notre objectif ici est de présenter les situations dans lesquelles la méthode de structuration spatiale peut apporter une aide à la compréhension de la répartition des vestiges au sol. Ce travail fait suite à celui d'A.-M. Christien (Christien et Bocquet, 1993) qui avait réalisé une telle approche sur une zone test réduite. Nous en avons présenté les principes et exposé leurs applications sur le site d'Egolzwil 3. Le cas des Baigneurs poursuit d'autres objectifs archéologiques. L'organisation générale villageoise est, certes, recherchée, mais aussi l'organisation 
interne des cellules architecturales repérées ou non par la dendrochronologie. Comment l'espace intérieur est-il segmenté ? Existe-t'il des espaces spécialisés repérables au sein des maisons? Lesquels ? En quoi et pourquoi ?

Les principaux obstacles à cette compréhension sont les plans des pieux, pas toujours lisibles en raison des durées variables des occupations humaines, des réfections nécessaires et des reconstructions. Par ailleurs, l'existence de structures en bois blanc est supposée. Enfin, à l'instar d'autres sites lacustres néolithiques, les nappes de vestiges y sont très diffuses.

Les conditions d'exploitation du gisement offrent un cas privilégié pour mener une étude à partir de la reconnaissance des structures horizontales au sol. En effet, tous les vestiges repérés, minéraux ou organiques, sont relevés par triangles de fouille (unités du carroyage en fouille subaquatique), soit par unités de surface de 0,43 mètres carrés. Tous les vestiges remontés sont relevés selon un double système de comptage/pesage et ce, pour toutes les unités de fouille ${ }^{3}$. Nous avons conservé douze catégories de vestiges : les gros éclats (Gé), les petits éclats (Pé), la céramique fine $(C f)$, la céramique grossière $(\mathrm{Cg})$, les quartzites brisés (Qua), les quartzites entiers (Cai), les os calcinés (Osc), les os non calcinés (Osnc), les boulettes d'argile (Bou), les coprolithes (Copr), les graines (Gr) et les fruits (Fr) (tab. 4).

Les nappes de vestiges, bien que diffuses, se sont formées en un temps bref (à peine plus de 25 années d'occupation reconnue sur le site). De plus, les hommes ont déplacé le village pour s'éloigner du lac lors d'une période plus humide. Les reconstructions sur un même espace sont donc limitées. Appréhender la disposition au sol des vestiges pour répondre à ces interrogations semble une piste intéressante à exploiter.

L'analyse est menée exactement selon les mêmes modalités que celle réalisée sur Egolzwil 3. Il ressort de l'analyse des étapes 1 à 5 ( $c f$. fig. 6) que les associations de vestiges sur le site s'effectuent suivant la richesse; mais les espaces riches en une catégorie de vestiges le sont en tout types de vestiges, d'où la difficulté d'observer des structures par plans de répartition. Sur l'axe 2 de l'analyse factorielle, se situent, d'une part, en positif, les modalités pauvres et, d'autre part, en négatif, les modalités riches. Ainsi, même si les unités riches comportent l'ensemble du panel des vestiges, les proportions de chaque vestige en sont variables. La classification ascendante hiérarchique montre que chaque classe se localise dans des zones bien précises et, nombre d'entre elles sont récurrentes d'une structure à une autre. Pour aider à l'interprétation que nous proposons ici, nous nous sommes appuyés, outres les indices statutaires sur les aspects qualitatifs. Comme indice à la compréhension de l'organisation du village complémentaires aux analyses statistiques, nous nous appuierons sur les structures architecturales clairement reconnues, pour cette occupation, par dendrochronologie (tab. 5).

Du point de vue de l'organisation générale du village, (cf. fig. $7 \& 8$ ) la station dans la partie étudiée s'étend exclusivement à l'intérieur de la palissade et s'articule autour d'une cour centrale qui se démarque des autres espaces exempts de vestiges (tels que les ruelles) par un empierrement massif. En effet, la classe 7 qui s'y localise, se caractérise par de grandes quantités d'os non calcinés et de quartzites brisés (utilisées comme pierres de chauffe). Ces deux types de restes ont dû volontairement être déposés-là afin de solidifier le sol en cas de forte humidité (pluies, inondations...) ou de piétinements intensifs.

Les animaux (de petite taille) devaient être parqués entre la maison 1 et le lac, espace où ils pouvaient facilement s'abreuver. Contrairement à ce que peut laisser voir le plan, la nappe de coprolithes (classe 5) ne s'étend pas sous les maisons mais empiète seulement légèrement sur les maisons car les reconstructions sont plus étroites que celles de la première phase.

L'organisation intérieure des unités domestiques se caractérise, d'une habitation à une autre, par des constantes d'assemblage de restes : classe 8 autour des foyers, classe 10 sous les auvents, classe 2 au niveau

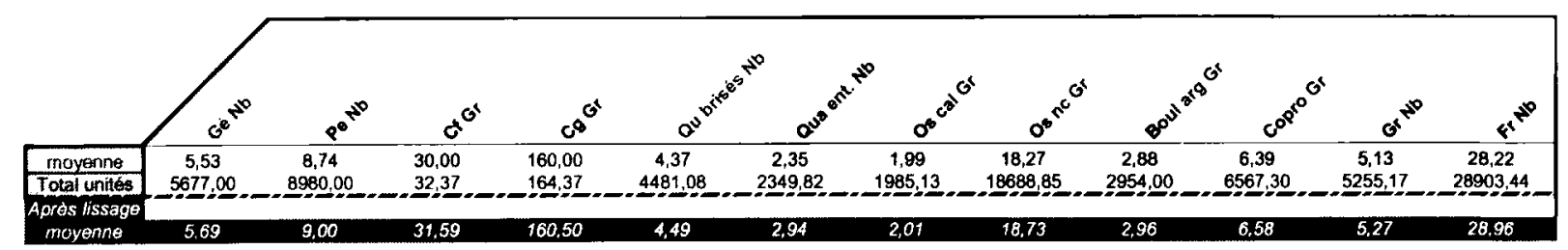

Tableau 4 : Résumés statistiques des catégories de témoins du site de Charavines les Baigneurs (B3)

Table 4: Statistical summary of remains categories of the site of Charavines les Baigneurs (B3) site.

\begin{tabular}{|c|c|c|c|c|c|c|c|c|c|c|}
\hline Chasse & $\overline{1}$ & 2 & 3 & 4 & 5 & 6 & 7 & 8 & 9 & 10 \\
\hline $\begin{array}{c}\text { Vestiges } \\
\text { principatux }\end{array}$ & $\begin{array}{l}\text { I:xempts de } \\
\text { vestiges } \\
\text { particuliers }\end{array}$ & Graines & $\begin{array}{c}\text { Fruml ()s } \\
\text { calcinés Gros } \\
\text { éclats }\end{array}$ & $\begin{array}{l}\text { Quartites } \\
\text { entiers }\end{array}$ & $\begin{array}{l}\text { Coprolithes } \\
\text { Céra grossière }\end{array}$ & $\begin{array}{l}\text { Boulettes } \\
\text { d'argiles }\end{array}$ & $\begin{array}{c}\text { (गs non calctnes } \\
\text { Quartaites } \\
\text { brisés }\end{array}$ & $\begin{array}{c}\text { Céramique: } \\
\text { grossière Céra } \\
\text { fine }\end{array}$ & $\begin{array}{l}\text { Peuts eclats } \\
\text { Gros éclats }\end{array}$ & $\begin{array}{l}\text { (3) not } \\
\text { calcines } \\
\text { Quartzites } \\
\text { brisés Os } \\
\text { calcinés }\end{array}$ \\
\hline $\begin{array}{c}\text { Vestiges } \\
\text { secondaires }\end{array}$ & & $\begin{array}{l}\text { Petits éclats } \\
\text { Coprolithes } \\
\text { Quartites }\end{array}$ & $\begin{array}{l}\text { Céra fine } \\
\text { Céra grossière } \\
\text { Petits éclats }\end{array}$ & $\begin{array}{c}\text { Os non } \\
\text { calcinés } \\
\text { Quartzites }\end{array}$ & $\begin{array}{c}\text { Qua. brisés } \\
\text { Graines Cérn } \\
\text { fine }\end{array}$ & & $\begin{array}{l}\text { Céra grossière } \\
\text { Qua. entiers } \\
\text { Céra fine }\end{array}$ & $\begin{array}{l}\text { Quartites } \\
\text { Gros éclats } \\
\text { Os calcinés } \\
\text { Coprolithes } \\
\text { Petts éclats } \\
\text { Fruits }\end{array}$ & Céra grossière & $\begin{array}{l}\text { Gros éclats } \\
\text { Petits éclats } \\
\text { Graines Céra } \\
\text { grossière Frnits }\end{array}$ \\
\hline
\end{tabular}

Tableau 5 : Composition des classes automatiques issues de la classification ascendante hićrarchique de Charavines les Baigncurs (B3).

Table 5: Final results of multidimensional methods authorizing us to group remains categories of Charavines les Baigneurs (B3). 3 Nous tenons à ce propos à citer M. A. Bocquet, directeur des fouilles de Charavines et le CDPA qui ont réalisé cette base informatique ct à remercicr
en particulier M. A. Bocquet de l'avoir misc à notre disposition. 


\section{Analyse en composantes principales}
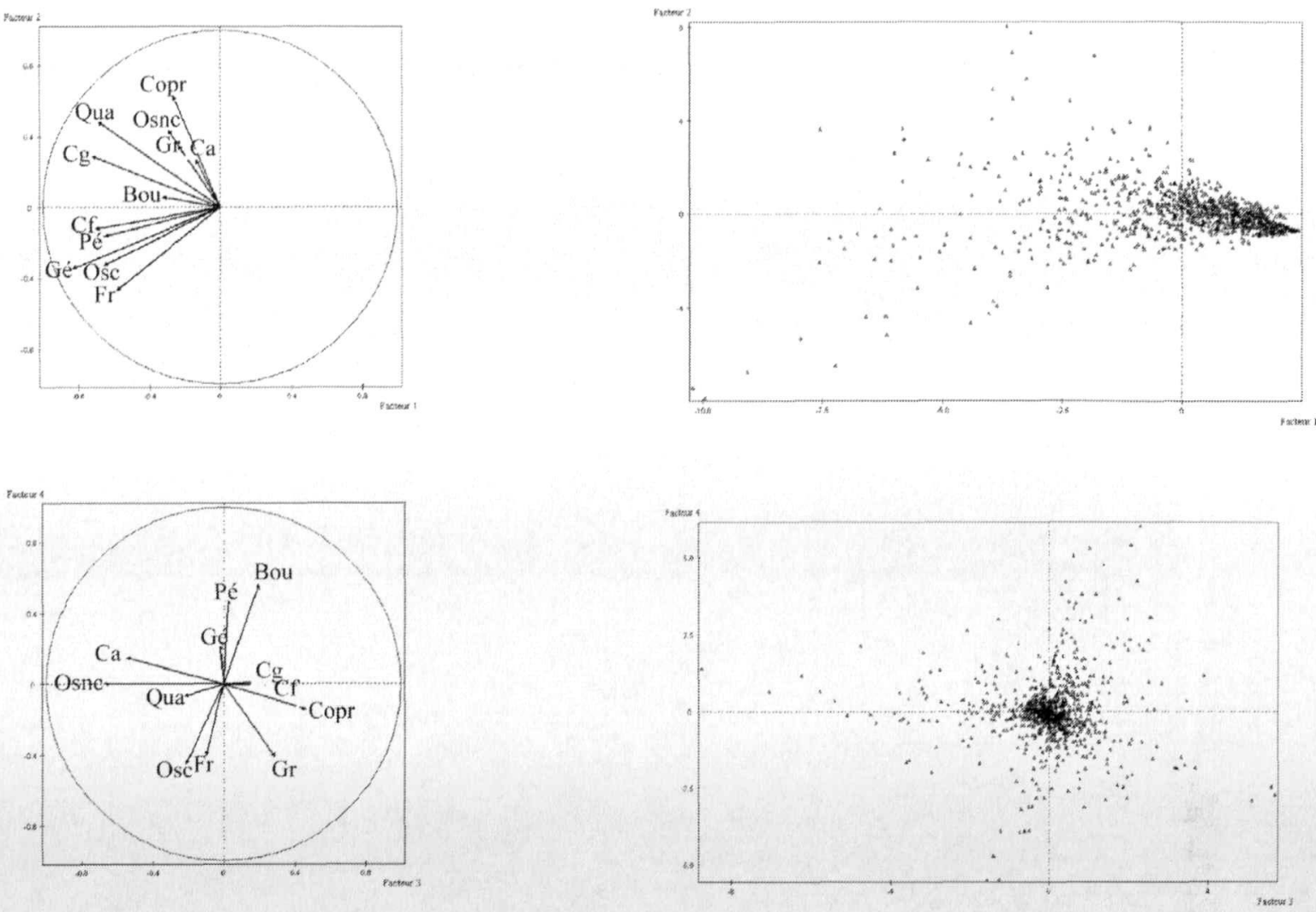

\section{Classification ascendante hiérarchique}
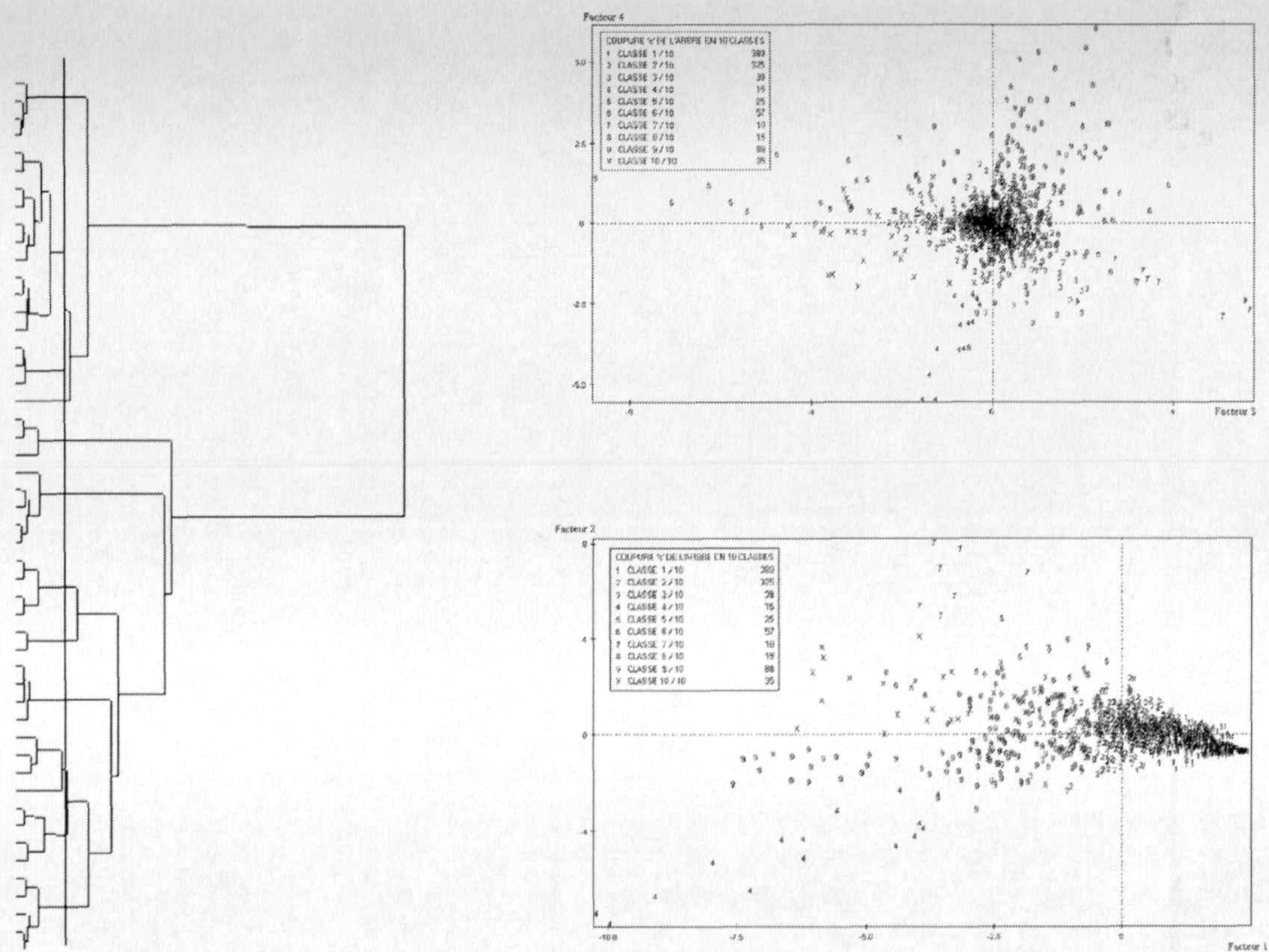

Figure 6 : Résultats graphiques de l'analyse en composantes principales de Charavines-les-Baigneurs. Figure 6: Graphical results solved by multidimensional methods of the site of Charavines les Baigneurs. 


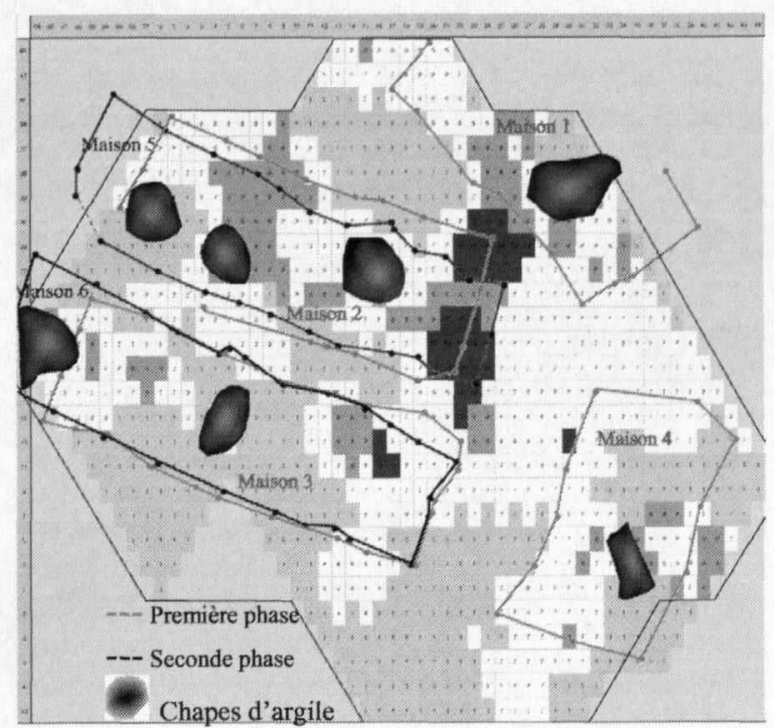

Figure 7 : Découpage en classes reporté en plan sur la station de Charavines-les-Baigneurs. Figure 7: Statistical results reported in a map on the site of Charavines les Baigneurs.

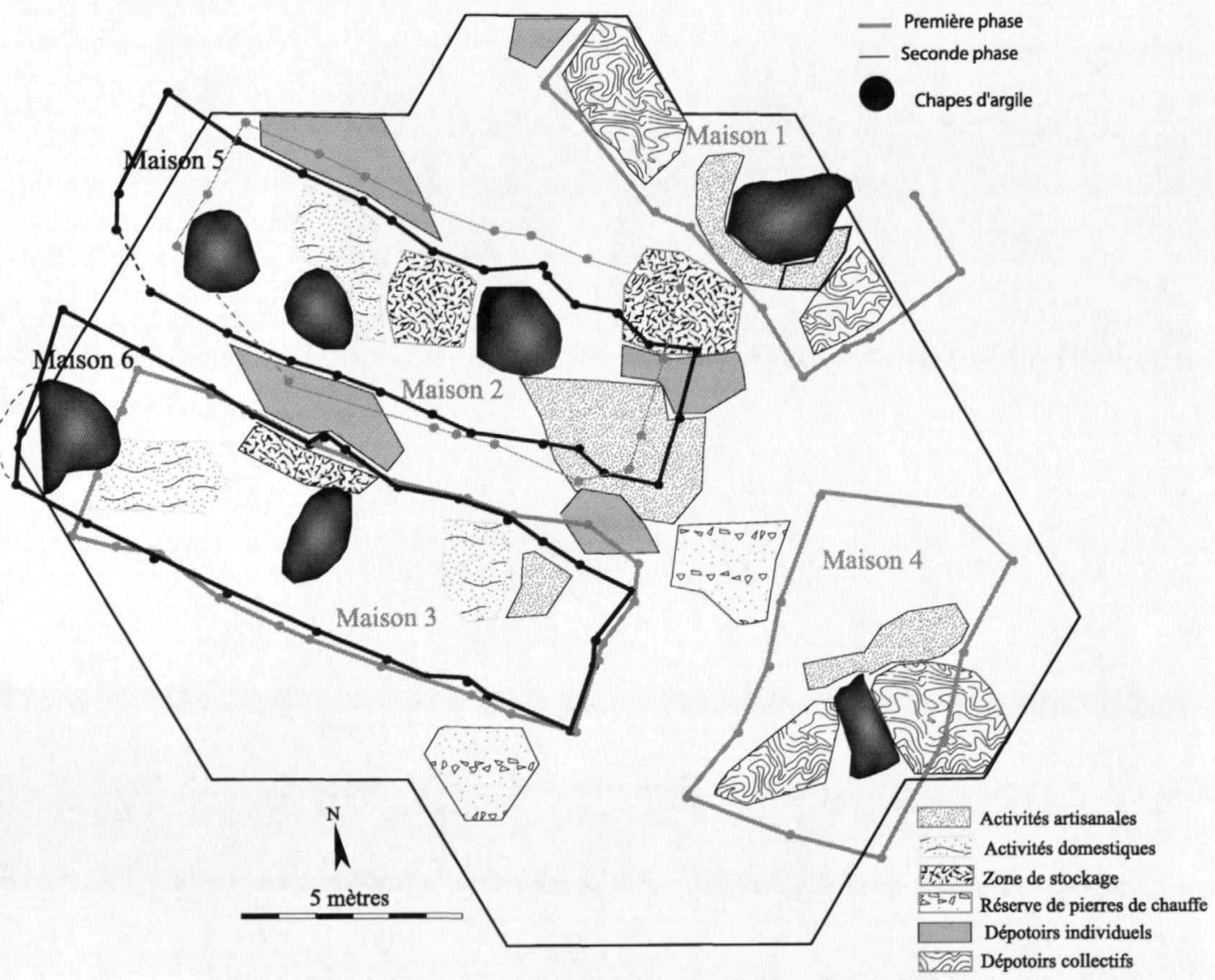

Figure 8 : Bilan de l'organisation spatiale de la station de Charavines-les-Baigneurs. Figure 8: Synthesis of the spatial organisation of the site of Charavines les Baigneurs.

d'ouvertures latérales supposées en raison des nappes étendues de vestiges sur le côté des maisons et des faibles quantités de rejets aux abords directs des foyers... La maison 3 présente cette même répartition des assemblages de restes, bien que les débris retrouvés y soient moins nombreux, en raison d'une situation topographique plus élevée la soumettant à une plus forte érosion. L'organisation des maisons semble tripartite.

L'avant des maisons, seulement couvert par un auvent (présence de deux travées avec seulement 3 alignements de poteaux), concentre l'essentiel des vestiges : os calcinés ou non, quartzites... (classe 10). Il doit abriter plusieurs activités allant de l'exploitation des matières premières pour les transformer à la fabrication des outils : taille du silex, filage de fibres textiles (grand nombre de fusaïoles et quelques fuseaux...). Ces activités nécessitent soit de la lumière, soit un grand espace, soit une aération (pour les besoins en oxygène du feu par exemple, pour éliminer des odeurs pénétrantes, et éventuellement conjointement, plusieurs de ces points). 
En entrant dans la partie des maisons comportant des parois, on trouve le foyer central de l'habitation. Celuici centralise les activités liées au domaine culinaire. Les vestiges montrent des activités allant du stockage et de la cuisson des aliments (présence de vase de stockage ou de préparation alimentaire en tonneau et cylindriques) à leur consommation (vases en calotte, globuleux, tronconiques...). Le stockage s'effectue derrière le foyer, vraisemblablement vers l'arrière de la maison, car c'est un espace de moindre circulation ( $c f$. résultats de l'analyse des correspondances). La préparation dont on retrouve les rejets de l'autre côté de la paroi a pu être réalisée à proximité d'ouvertures latérales (repérées à partir des vestiges architecturaux et de l'observation de plusieurs amoncellements de vestiges perpendiculaires à chaque maison) et la consommation effectuée vers l'avant et à proximité des foyers qui apportent chaleur et lumière. Dans cette direction, la présence de nombreux éclats confirme ce besoin d'outillage (découpe...).

Enfin, la partie arrière des maisons se révèle particulièrement pauvre. D'après l'analyse des vestiges, cet espace aurait pu être destiné au couchage ; cependant des traces de nattes semblent exister près des foyers centraux qui offrent une source de chaleur, ce qui infirme l'hypothèse précédente. Toutefois, la propension des nattes et des litières en sapin à s'enflammer rapidement, ainsi que le fait que l'ouverture latérale soit située à proximité des foyers est paradoxale, laissant la question ouverte.

Une fois devenus trop nombreux, les rejets déversés par les ouvertures latérales, sont secondairement déplacés durant une phase d'occupation postérieure où le cœur du village se serait déplacé, plus à l'abri de l'humidité (observations dendrochronologiques). Ces rejets auraient été apportés, comme l'attestent de nombreux remontages, vers un dépotoir collectif, au nord du village, sur les restes des maisons antérieures, telles la 1 ou la 4 . L'étude des stratigraphies ne permet pas de trancher pour assurer que la classe 9 se soit déposée postérieurement aux autres classes représentatives de l'intérieur des habitations. Il semble toutefois qu'elle caractérise les maisons abandonnées durant la deuxième phase d'occupation du village, idée corroborée également par la dendrochronologie. La classe 6 montre également cette succession avec les boulettes d'argile retrouvées sur les foyers, à l'exclusion de tout autre type de restes qui montrent que les foyers doivent être encore utilisés impliquant le fonctionnement des unités domestiques lors du départ des villageois.

Enfin, au niveau de l'avant des maisons 2 et 3, on peut trouver des tas clairement délimités de quartzites entiers (classe 4). Ces probables réserves de pierres de chauffe sont un indice supplémentaire pour confirmer la chronologie des constructions du village aussi établie par la dendrochronologie.

On remarque que toutes les étapes nécessaires à la vie quotidienne semblent être représentées dans chaque unité domestique : chauffage, cuisson, préparation et consommation des repas, ainsi que utilisation et fabrication des outils amènent à considérer ces structures comme des cellules domestiques élémentaires.

Ces analyses spatiales montrent pour les deux stations un système de rejet, système observable grâce aux obser- vations conjointes des catégories de vestiges, depuis les structures architecturales directement à l'intérieur du village. De l'espace intérieur des structures, il ressort une organisation similaire à travers les deux villages. En effet, les types d'activités génériques : «artisanales », domestiques... prennent place dans les espaces intérieurs aux caractéristiques similaires, aux abords des foyers, sous les auvents... Ces activités peuvent toutefois être de nature différentes, ainsi les activités " artisanales " vont de la production d'outils à leur utilisation : raclage des végétaux, travail des peaux ( $c f$. analyses tracéologiques), mouture, taille de silex... Les abords directs du foyer central des habitations sont le lieu des activités domestiques liées essentiellement aux activités culinaires comme le stockage, la préparation (faune et macro restes) ou la cuisson (vases de cuisson) et la consommation des aliments (vases de plus petite taille, restes fauniques calcinés...). L'étude conjointe de l'organisation spatiale des activités montre ainsi des systèmes similaires d'organisation des activités dans les unités domestiques et des rejets sur les stations d'Egolzwil 3 et de Charavines-les-Baigneurs.

\section{Conclusion}

La reconnaissance de l'organisation de l'espace en milieu lacustre permet d'aborder des thèmes rarement envisageables comme l'organisation des activités à l'intérieur de l'unité domestique (économie domestiques ou spécialisées) en dehors de ces conditions privilégiées. Toutefois, nous avons vu la nécessité de trouver les outils d'analyse adaptés aux problèmes spécifiques soulevés par ce milieu. La mise en œuvre de méthodes multidimensionnelles a permis de mettre au jour des structures spatiales, amas ou éparpillements (zones de travail « artisanal » par exemple) avec une indépendance totale vis-àvis des structures architecturales. L'emploi de classifications permet de définir des comportements de variables et des témoins présents dans chaque triangle de fouille et, ainsi, d'observer des associations et des oppositions de certaines catégories de témoins de natures différentes. En cela, l'utilisation d'outils multidimensionnels pour mener une approche spatiale est novatrice.

Les analyses ont permis de proposer des modèles d'organisation spatiale des villages d'Egolzwil 3 et de Charavines-les-Baigneurs B3. Les indices socio-économiques des communautés proposés sur la base des distributions de vestiges dans l'espace villageois, montrent des oppositions spatiales : intérieur/extérieur, activités « artisanales »/ domestiques et lieux de vie / lieux de rejets. Pour cela l'analyse doit alors prendre en compte les aspects qualitatifs des matériaux : typologie fine des catégories de matériel, aspects technologiques relatifs à la chaîne opératoire, témoins d'activité « spécialisée ", fragmentation du mobilier...

Une fois cette structuration reconnue, il est nécessaire d'affiner la définition de chaque aire d'activité pour identifier les activités qui y étaient pratiquées, des indices de fonctionnement entre les unités architecturales en recherchant des attributs spécifiques par catégories de témoins peuvent également être isolés. Ces attributs peuvent être interprétés en terme de fonctionnement 
des cellules élémentaires et du fait de fonctionnement villageois. Simultanément, il faudrait multiplier ces approches anthropologiques de l'espace avec ces outils vers d'autres sites aux structurations spatiales particulières, afin de pouvoir saisir l'origine de ces organisations : facteurs environnementaux, culturels, identitaires...

Une recherche généralisée, par la mise en cuvre de méthodes appropriées, des organisations villageoises spatiales et structurelles devrait pouvoir apporter des indices d'une nature nouvelle à nos connaissances sur le mode de vie et les processus identitaires des communautés néolithiques.

\section{Bibliographie}

ARBOGAST, R.-M., BEUGNIER, V., DELATTRE, N., GILIGNY, F., Maltre, A., PETRequin, A.-M. \& PETrequiN, P., 1997 - La répartition des témoins et le fonctionnement de la cellule domestique. In P. Pétrequin (dir.) les sites littoraux néolithiques de Clairvaux-les-lacs et de Chalain (Jura) III. Edition de la Maison des Sciences de l'Homme, Paris.

BINFORD, L.R., 1978 - Dimensional Analysis of Behaviour and Site Structure: Leaming from Eskimo Hunting Stand, American Antiquity, $43,330-361$.

BOCQUET, A., 1994 - Charavincs il y a 5000 ans, Les dossiers de l'archéologie, $\mathrm{n}^{\circ} 199$.

BORDES, F., 1975 - Sur la notion de sol d'habitat en préhistoirc palćolithique. In Bulletin de la Société Préhistorique Française, tome 72 $n^{\circ} 5,139-145$.

CHRISTIEN, A.-M. \& BOCQUET, A., 1993 - L'organisation spatiale de la station néolithique de Charavines-les-Baigneurs (Isc̀re), In J.C. Blanchet, A. Bulard, C. Constantin, D. Mordant et J. Tarrête, le néolithique au quotidien, Actes du 16 c̀me Colloque Interrégional sur le Nćolithique, Paris, 5-6 Nov. 1989. Documents d'Anchéologie Française $n^{\circ} 39,63-71$.

CLARK, P.J. \& EVANS, F.C., 1954 - Distance to neighbour as nearest as mcasure of spatial relationships in populations. Ecology $n^{\circ} 55,445-453$.

COLOMER, A., COUlarou, J. \& GUTHERZ, X., 1990 - Boussargues (Argelliers, Hérault), un habitat ceinturé chalcolithique : les fouilles du sectcur ouest. Documents d'Archéologie Française $n^{\circ} 24$, Editions de la Maison des Sciences de l'Homme, Paris.

DEMOULE, J.P, GILIGNY, F., LEHÖERFF, A. \& SCHNAPP, A., 2002 - Guide des méthodes de l'archéologie. ed. La Découvcrtc \& Syros, Coll. Repères, Paris.

DJINDJIAN, F., 1988 - Improvement in intrasitc spatial analysis techniques, Computer and quantitative methods in archacology, Oxford Bar International series 446, Ratz, 95-106.

DJINDJIAN, F., 1999 - L'analysc spatiale de l'habitat : un ctat de l'art. Archeologia e calculatori $n^{\circ} 10,17-32$

DURKHEIM, E., 1937 - Les règles de la méthode sociologique, préface 2ème édition. Presses Universitaires de France, Paris.

KROLLL \& PRICE, D., 1991 - The interpretation of archaeological spatial Panterning. New York Press.

LEAKEY, M.D., 1971 - Oldwai Gorge, Vol. 3. Excavation in Beds I and II, 1960-1963, Cambridge University Press, Cambridge.

LEROI-GOURHAN, A. \& BREZILLON, M., 1966 - L'habitation magdalénienne $n^{\circ} 1$ de Pincevent près de Montereau (Seine-ctMarne). Gallia Préhistoire, IX-2, 263-385.
LEROI-GOURHAN, A. \& BREZILLON, M., 1972 - Fouilles dc Pincevent : cssai d'analyse d'un habitat magdalénien : la section 36 , Gallia Préhistoire, supplément 7, CNRS éditions, Paris.

MAGNY, M., 1978 - Dynamique des dépôts lacustres et les stations littorales du Grand Lac de Clairvaux (Jura). Paris : Editions du CNRS (Notes et monographies techniques, II), $222 \mathrm{p}$.

MAGNY, M., 1993 - Une nouvelle mise en perspective des sites archéologiques lacustres : les fluctuations holocénes des lacs jurassiens et subalpins. Gallia préhistoire $n^{\circ} 35$ CNRS éditions, Paris, 253-282.

MONNIER, J.-L., PETREQUIN, P. \& RICHARD, A., 1991 - Construire une maison en $3000 \mathrm{cN}$. J.-C., Le lac de Chalain au néolithique. Ed. Errance Paris, $75 \mathrm{p}$.

PETREQUIN, P., 1981 - La grotte des planches prés-Arbois (Jura) Proto-cortaillod et âge du bronze final. Thèse de doctorat d'état de l'université de Franche-Comté faculté des lettres.

PETREQUIN, A.-M. \& PETREQUIN, P., 1984 - Habitat lacustre de Bénin, une approche ethno-archéologique. Editions Recherche sur les Civilisations, mémoire $n^{\circ} 39$, Paris.

PETREQUiN, P., ARboGAST, R.-M., BOURQUiN-MIGNOT, C., DUPLAIX, A., MARTINEAU, R. \& PETREQUiN, A.-M., 2002 - Le mythe de la stabilité : déséquilibres et réajustements d'une communauté agricole néolithique dans le Jura français, du $32 \mathrm{èmc} \mathrm{au}$ 30 ème siècle av. J.-C. In H. Richard. ct A. Vignot A. (dir.), Actes du colloque international de Besançon Equilibres et ruptures dans les écosystèmes churant les 20 derniers millénaires en Europe de l'Ouest, Sept. 2000. Besançon, Presses Universitaires Franc-Comtoises, 175190.

SCHIFFER, M., 1976 - Behavioural archaeology. New York, Academic Press.

TARDIEU, C., 2000 - Analyse spatiale de Charavines-les-Baigneurs Etude et comparaison des occupations, Mémoire de D.E.A. Anthropologic-Ethnologic-Préhistoire, Université de Paris I.

TARDIEU, C., 2002 - Application d'une méthode d'analyse spatiale au village lacustre néolithique de Charavines-les-Baigneurs (Isère, Francc), Bulletin de la Société Préhistorique Française tome $99 n^{\circ} 2$, Paris.

TARDIEU, C., 2004 - Espaces de vie et identités au Nćolithique : approche spatiale des communautés lacustres du domaine circumalpin, thèse de doctorat sous la direction de $\mathrm{S}$. van des Leeuw, Université de Paris I.

VAN NOTTEN, F. et al., 1978 - Les chasseurs du Meer, de Temple, Brugge.

WHALLON, R., 1973 - Spatial analysis of Palacolithic occupations area : the present problem and the "functional argument". In C. Renfrcw (Ed.) the explanation of culture change : models in prehistory, London, Duckworth.

WHALLON, R., 1974 - Spatial analysis of occupation floors: the application of nearest neighbour analysis. American Antiquity $n^{\circ} 39$, 16-34.

WHALLON, R., 1984 - Unconstrained clustering for the analysis of spatial distributions in archacology. In $\mathrm{H}$. Hictla (dir.) Intrasite spatial analysis in archaeology, Cambridge university Press, New York, 242-277.

WYSS, R., 1994 - Steinzeitliche Bauern auf der Suche nach neuen Lebensformen Egolzwil 3 und die Egolzwiler Kultur 1/Die Funde. Archeaologische Forschungen, Schweizerisches Landesmuseum, Zürich.

WYSS, R., 1996 - Steinzeitliche Bauern auf der Suche nach neuen Lebensformen Egolzwil 3 und die Egolzwiler Kultur 2/Dir Grabungsergebnisse. Archeaologische Forschungen, Schweizerisches Landesmuseum, Zürich.

YELLEN, J.E., 1977 - Archaeological approaches to the present: Models for reconstructing the past. New York, Academic Press. 